\title{
ON THE BOUNDEDNESS AND COMPACTNESS OF WEIGHTED GREEN OPERATORS OF SECOND-ORDER ELLIPTIC OPERATORS
}

\author{
YEHUDA PINCHOVER
}

\begin{abstract}
For a given second-order linear elliptic operator $L$ which admits a positive minimal Green function, and a given positive weight function $W$, we introduce a family of weighted Lebesgue spaces $L^{p}\left(\phi_{p}\right)$ with their dual spaces, where $1 \leq p \leq \infty$. We study some fundamental properties of the corresponding (weighted) Green operators on these spaces. In particular, we prove that these Green operators are bounded on $L^{p}\left(\phi_{p}\right)$ for any $1 \leq p \leq \infty$ with a uniform bound. We study the existence of a principal eigenfunction for these operators in these spaces, and the simplicity of the corresponding principal eigenvalue. We also show that such a Green operator is a resolvent of a densely defined closed operator which is equal to $\left(-W^{-1}\right) L$ on $C_{0}^{\infty}$, and that this closed operator generates a strongly continuous contraction semigroup. Finally, we prove that if $W$ is a (semi)small perturbation of $L$, then for any $1 \leq p \leq \infty$, the associated Green operator is compact on $L^{p}\left(\phi_{p}\right)$, and the corresponding spectrum is $p$-independent.
\end{abstract}

2000 Mathematics Subject Classification. Primary 35J08; Secondary 35B09, 35J15, 35P05, 47D06.

Keywords. Green function, ground state, Liouville theorem, positive solution, principal eigenvalue, small perturbation.

\section{INTRODUCTION}

Positive (Dirichlet) Green functions of second-order linear elliptic operators with real coefficients and their induced integral operators are among the most important building blocks of the elliptic theory for such operators, and in particular, for the qualitative theory of positive solutions of the corresponding homogeneous equations. In many problems, and in particular in the study of positive solutions, the underling topology is the open compact topology, i.e. the topology of locally compact convergence (e.g., in the Martin boundary theory, in the study of the heat kernel, and in criticality theory). On the other hand, when dealing with spectral theory of such operators, in the study of semigroups generated by such operators, or in

Dedicated to Professor Pavel Exner on the occasion of his 70th birthday. The author is grateful to Professor M. Cwikel and the late professor V. Liskevich for valuable discussions. This research was supported by the Israel Science Foundation (grants No. 963/11) founded by the Israel Academy of Sciences and Humanities. 
the study of well-posedness of boundary value problems, one should usually specify a relevant Banach space. Frequently, one takes one of the classical Lebesgue spaces as the underlying space, but a priori, it is not clear why these spaces are the appropriate functional spaces to study various elliptic problems for a specific operator in a given domain.

In the present paper, we introduce for a given second-order linear elliptic operator $L$ which is defined on a noncompact manifold $\Omega$ and admits a positive Green function, and for a given positive weight function $W$, a family of weighted Lebesgue spaces $L^{p}\left(\phi_{p}\right)$, where $1 \leq p \leq \infty$. The weight $\phi_{p}$ is given by

$$
\phi_{p}:=\phi^{-1}(\phi W \tilde{\phi})^{1 / p},
$$

where $\phi$, (resp. $\tilde{\phi})$ is a fixed positive solution of the equation $(L-\mu W) u=0$ (resp. $\left.\left(L^{\star}-\mu W\right) u=0\right)$ in $\Omega$, and $L^{\star}$ is the formal adjoint of $L$ (see Section 2 for a detailed discussion on these spaces, and also for the needed terminology and some preliminary results).

Clearly, if the positive Liouville theorem holds for $L-\mu W$ and $L^{\star}-\mu W$ in $\Omega$, then $L^{p}\left(\phi_{p}\right)$ is uniquely defined. In addition, $L^{1}\left(\phi_{1}\right)$ is always independent of $\phi$ while $L^{\infty}\left(\phi_{\infty}\right)$ is independent of $\tilde{\phi}$ and $W$. In [28], $L^{\infty}\left(\phi_{\infty}\right)$ was introduced, and some properties of weighted Green operators on $L^{\infty}\left(\phi_{\infty}\right)$ were studied. Moreover, if $L$ is symmetric, then one may choose $\tilde{\phi}=\phi$, and then $L^{2}\left(\phi_{2}\right)=L^{2}(\Omega, W)$, and hence, this space is $\phi$ independent. We also note that $\left\{L^{p}\left(\phi_{p}\right)\right\}_{p \geq 1}$ is a family of real interpolation spaces. The latter observation is used to prove several results of the paper.

The aim of the present paper is to study some fundamental properties of the induced weighted Green operators on these weighted Lebesgue spaces. In particular, we prove in Section 3 that the corresponding weighted Green operator is bounded on $L^{p}\left(\phi_{p}\right)$ for any $1 \leq p \leq \infty$ with a bound independent of $p, \phi$, and $\tilde{\phi}$. In Section 4, we study the existence and uniqueness of a principal eigenfunction for these weighted Green operators on $L^{p}\left(\phi_{p}\right)$, and the simplicity of the corresponding principal eigenvalue for $1 \leq p \leq \infty$.

Next, we show in Section 5 that for $1 \leq p<\infty$, the weighted Green operator is a resolvent of a densely defined closed operator $A_{p}$ such that $A_{p}=$ $-W^{-1} L$ on $C_{0}^{\infty}(\Omega)$. It turns out that under some further assumptions, $A_{p}$ generates a strongly continuous contraction semigroup. Finally, in Section 6 we prove that if $W$ is a (semi)small perturbation of $L$ in $\Omega$, then for any $1 \leq$ $p \leq \infty$, the associated weighted Green operator is compact on $L^{p}\left(\phi_{p}\right)$, and the corresponding spectrum is $p$-independent. We note that if in addition $L$ is symmetric, then it follows from [28] that for any $k \geq 1$ the quotient $\phi_{k} / \phi$ has a continuous extension up to the Martin boundary of the pair $(\Omega, L)$, where $\phi$ is the ground state of $L$ with a principal eigenvalue $\lambda_{0}$, and $\phi_{k}$ is a weighted eigenfunction in $L^{2}(\Omega, W \mathrm{~d} \nu)$. It follows from the $p$-independence of the spectrum that in fact, $\phi, \phi_{k} \in L^{p}\left(\phi_{p}\right)$, for all $1 \leq p \leq \infty$. 
The problem of the $p$-independence of the spectrum of generators of semigroups on $L^{p}$ was raised by B. Simon in 33] for Schrödinger semigroups in $\mathbb{R}^{d}$ and has been studied in many papers, see for example $[4,10,11,15,18$, 19, 31, 32, 33, 34 and references therein.

Various sufficient conditions that guarantee that $L$ has a pure pointspectrum in certain spaces is given for example in [6, 16, 20] and references therein.

\section{Preliminaries}

2.1. Elliptic operators and positive solutions. Let $\Omega$ be a domain in $\mathbb{R}^{d}$, or more generally, a noncompact connected $C^{2}$-smooth Riemannian manifold of dimension $d$. By a positive function we mean a strictly positive function. We assume that $\nu$ is a positive measure on $\Omega$, satisfying $\mathrm{d} \nu=f$ vol with $f$ a positive measurable function; vol being the volume form of $\Omega$ (which is just the Lebesgue measure in the case of a domain $\Omega$ in $\mathbb{R}^{d}$ ). We write $\Omega_{1} \Subset \Omega_{2}$ if $\Omega_{2}$ is open, $\overline{\Omega_{1}}$ is compact and $\overline{\Omega_{1}} \subset \Omega_{2}$. Denote by $B\left(x_{0}, \delta\right)$ the open ball of radius $\delta>0$ centered at $x_{0}$. Let 1 be the constant function on $\Omega$ taking at any point $x \in \Omega$ the value 1 . For a matrix $A(x)=\left[a^{i j}(x)\right]$ and a vector field $b(x)=\left(b^{j}(x)\right)$ we denote

$(A(x) \xi)^{i}:=\sum_{j=1}^{d} a^{i j}(x) \xi_{j}, \quad b(x) \cdot \xi:=\sum_{j=1}^{d} b^{j}(x) \xi_{j}$, where $\xi=\left(\xi_{1}, \ldots, \xi_{d}\right) \in \mathbb{R}^{d}$.

We associate to $\Omega$ a fixed exhaustion $\left\{\Omega_{n}\right\}_{n=1}^{\infty}$, i.e., a sequence of smooth, relatively compact subdomains such that $\Omega_{1} \neq \emptyset, \Omega_{k} \Subset \Omega_{k+1}$ and $\cup_{k=1}^{\infty} \Omega_{k}=$ $\Omega$. For every $k \geq 1$, we denote $\Omega_{k}^{\star}=\Omega \backslash \overline{\Omega_{k}}$.

Let $L$ be a linear, second-order, elliptic operator defined on $\Omega$. We assume that the coefficients of $L$ are real, and that in any coordinate system $\left(U ; x_{1}, \ldots, x_{n}\right)$, the operator $L$ is of the divergence form

$$
L u:=-\operatorname{div}(A(x) \nabla u+u \tilde{b}(x))+b(x) \cdot \nabla u+c(x) u .
$$

Here, the minus divergence is the formal adjoint of the gradient with respect to the measure $\nu$.

We assume that for every $x \in \Omega$ the matrix $A(x):=\left[a^{i j}(x)\right]$ is symmetric, and the associated real quadratic form

$$
\xi \cdot A(x) \xi:=\sum_{i, j=1}^{d} \xi_{i} a^{i j}(x) \xi_{j} \quad \xi \in \mathbb{R}^{d}
$$

is positive definite. Moreover, throughout the paper it is assumed that $L$ is locally uniformly elliptic, and the coefficients of $L$ are real valued and locally sufficiently regular in $\Omega$. All our results hold for example when $L$ is of the form (2.1), and $A$ and $f$ are locally Hölder continuous, $b, \tilde{b} \in$ $L_{\text {loc }}^{p}\left(\Omega ; \mathbb{R}^{n}, \mathrm{~d} x\right)$, and $c \in L_{\text {loc }}^{p / 2}(\Omega ; \mathbb{R}, \mathrm{d} x)$ for some $p>d$. However it would be apparent from the proofs that any conditions that guarantee standard 
elliptic regularity theory are sufficient. By a potential defined in $\Omega$, we mean a function $V \in L_{\text {loc }}^{p / 2}(\Omega ; \mathbb{R}, \mathrm{d} x)$ for some $p>d$.

The formal adjoint $L^{\star}$ of the operator $L$ is defined on its natural space $L^{2}(\Omega, \mathrm{d} \nu)$. When $L$ is in divergence form (2.1) and $b=\tilde{b}$, the operator

$$
L u=-\operatorname{div}(A \nabla u+u b)+b \cdot \nabla u+c u,
$$

is symmetric in the space $L^{2}(\Omega, \mathrm{d} \nu)$. Throughout the paper, we call this setting the symmetric case. We note that if $L$ is symmetric and $b$ is smooth enough, then $L$ is in fact a Schrödinger-type operator of the form

$$
L u=-\operatorname{div}(A \nabla u)+V u, \quad \text { where } V:=(c-\operatorname{div} b) .
$$

By a solution $v$ of the equation $L u=0$ in $\Omega$, we mean $v \in W_{\text {loc }}^{1,2}(\Omega)$ that satisfies the equation $L u=0$ in $\Omega$ in the weak sense. Subsolutions and supersolutions are defined similarly. We denote the cone of all positive solutions of the equation $L u=0$ in $\Omega$ by $\mathcal{C}_{L}(\Omega)$.

Remark 2.1. We would like to point out that the theory of positive solutions of the equation $L u=0$ in $\Omega$ (the so-called criticality theory), and in particular the results of this paper, are also valid for the class of classical solutions of locally uniformly elliptic operators of the form

$$
L u=-\sum_{i, j=1}^{d} a^{i j}(x) \partial_{i} \partial_{j} u+b(x) \cdot \nabla u+c(x) u,
$$

with real and locally Hölder continuous coefficients, and for the class of strong solutions of locally uniformly elliptic operators of the form (2.3) with locally bounded coefficients (provided that the formal adjoint operator also satisfies the same assumptions). Nevertheless, for the sake of clarity, we prefer to present our results only for the class of weak solutions.

Fix a nonzero nonnegative potential $W$ defined in $\Omega$, and for $\lambda \in \mathbb{R}$ denote by $L_{\lambda}$ the elliptic operator $L-\lambda W$. Consider the (weighted) generalized principal eigenvalue of the operator $L$

$$
\lambda_{0}=\lambda_{0}(L, W, \Omega):=\sup \left\{\lambda \in \mathbb{R} \mid \mathcal{C}_{L_{\lambda}}(\Omega) \neq \emptyset\right\} .
$$

Note that if $\lambda_{0} \neq-\infty$ (as assumed throughout the present paper), then in fact, $\lambda_{0}=\max \left\{\lambda \in \mathbb{R} \mid \mathcal{C}_{L_{\lambda}}(\Omega) \neq \emptyset\right\}$.

If $\lambda_{0} \geq 0$, then for every $k \geq 1$ the Dirichlet Green function $G_{L}^{\Omega_{k}}(x, y)$ of the operator $L$ in $\Omega_{k}$ exists and is positive. By the generalized maximum principle, $\left\{G_{L}^{\Omega_{k}}(x, y)\right\}_{k=1}^{\infty}$ is an increasing sequence converging as $k \rightarrow \infty$ either to $G_{L}^{\Omega}(x, y)$, the positive minimal Green function of $L$ in $\Omega$, and then $L$ is said to be a subcritical operator in $\Omega$, or to infinity and in this case $L$ is critical in $\Omega$. If $\mathcal{C}_{L}(\Omega)=\emptyset$, then $L$ is supercritical in $\Omega$ [21, 24] (cf. [33]).

It follows that $L$ is critical (resp. subcritical) in $\Omega$, if and only if $L^{\star}$ is critical (resp. subcritical) in $\Omega$. Clearly, $L_{\lambda}$ is subcritical in $\Omega$ for every $\lambda \in\left(-\infty, \lambda_{0}\right)$, and supercritical for $\lambda>\lambda_{0}$. Furthermore, if $L$ is critical in 
$\Omega$, then $\mathcal{C}_{L}(\Omega)$ is a one-dimensional cone and any positive supersolution of the equation $L u=0$ in $\Omega$ is a solution. So, in the critical case, $\phi \in \mathcal{C}_{L}(\Omega)$ is uniquely defined (up to a multiplicative positive constant), and such $\phi$ is called the Agmon ground state of $L$ in $\Omega$.

Subcriticality is a stable property in the following sense. If $L$ is subcritical in $\Omega$ and $V$ is a potential with a compact support in $\Omega$, then there exists $\varepsilon>0$ such that $L-\mu V$ is subcritical, for all $|\mu|<\varepsilon[21,24$. On the other hand, if $L$ is critical in $\Omega$ and $V$ is a nonzero, nonnegative potential, then for any $\varepsilon>0$ the operator $L+\varepsilon V$ is subcritical and $L-\varepsilon V$ is supercritical in $\Omega$.

Definition 2.2 (Agmon [1]). Let $L$ be an elliptic operator of the form (2.1) defined in $\Omega$. A function $u \in \mathcal{C}_{L}\left(\Omega_{n}^{\star}\right)$ is said to be a positive solution of the equation $L u=0$ of minimal growth in a neighborhood of infinity in $\Omega$, if for any $k>n$ and any $v \in \overline{C\left(\Omega_{k}^{\star}\right)}$ which is a positive supersolution of the equation $L w=0$ in $\Omega_{k}^{\star}$, the inequality $u \leq v$ on $\partial \Omega_{k}$ implies that $u \leq v$ in $\Omega_{k}^{\star}$.

In the sequel, in order to simplify our terminology, we will call a positive minimal Green function - a Green function, an Agmon ground state - a ground state, and a positive solution of minimal growth in a neighborhood of infinity in $\Omega$, a positive solution of minimal growth in $\Omega$.

It turns out that if $L$ is subcritical in $\Omega$, then for any fixed $y \in \Omega$ the Green function $G_{L}^{\Omega}(\cdot, y)$ is a positive solution of the equation $L u=0$ in $\Omega \backslash\{y\}$ of minimal growth in $\Omega$. On the other hand, if $L$ is critical in $\Omega$, then the ground state $\phi$ is a (global) positive solution of the equation $L u=0$ in $\Omega$ of minimal growth in $\Omega$.

Fix a nonzero nonnegative potential $W$ defined in $\Omega$. Let $v$ and $\tilde{v}$ be positive solutions of the equations $L_{\mu} u=0$ and $L_{\mu}^{\star} u=0$ in $\Omega$, respectively, where $\mu \leq \lambda_{0}$. Then [26] for every $\lambda<\mu$ we have

$$
\begin{array}{ll}
\int_{\Omega} G_{L_{\lambda}}^{\Omega}(x, y) W(y) v(y) \mathrm{d} \nu(y) \leq \frac{v(x)}{\mu-\lambda} & \forall x \in \Omega, \\
\int_{\Omega} G_{L_{\lambda}}^{\Omega}(x, y) W(x) \tilde{v}(x) \mathrm{d} \nu(x) \leq \frac{\tilde{v}(y)}{\mu-\lambda} & \forall y \in \Omega .
\end{array}
$$

Moreover, in each of the inequalities in (2.5) either equality or strict inequality holds for all points in $\Omega$ and $\lambda<\mu$. If equality holds for $v$ (resp. $\tilde{v}$ ), then $v$ (resp. $\tilde{v}$ ) is said to be a positive invariant solution of the equation $L_{\mu} u=0$ (resp. $L_{\mu}^{\star} u=0$ ) in $\Omega$, or $\mu$-invariant solution of the operator $L$ (resp. $\left.L^{\star}\right)$ in $\Omega$.

Assume now that $L_{\lambda_{0}}$ is critical in $\Omega$ with $\lambda_{0} \in \mathbb{R}$, and let $\phi$ and $\tilde{\phi}$ be the ground states of $L_{\lambda_{0}}$ and $L_{\lambda_{0}}^{\star}$, respectively. Then [26, Theorem 2.1] $\phi$ and $\tilde{\phi}$ are positive invariant solutions of the equations $L_{\lambda_{0}} u=0$ and $L_{\lambda_{0}}^{\star} u=0$, 
respectively, Hence, for every $\lambda<\lambda_{0}$ we have

$$
\begin{aligned}
& \int_{\Omega} G_{L_{\lambda}}^{\Omega}(x, y) W(y) \phi(y) \mathrm{d} \nu(y)=\frac{\phi(x)}{\lambda_{0}-\lambda} \quad \forall x \in \Omega, \\
& \int_{\Omega} G_{L_{\lambda}}^{\Omega}(x, y) W(x) \tilde{\phi}(x) \mathrm{d} \nu(x)=\frac{\tilde{\phi}(y)}{\lambda_{0}-\lambda} \quad \forall y \in \Omega .
\end{aligned}
$$

Assume further that $W$ is a positive function. If $\phi \tilde{\phi} \in L^{1}(\Omega, W \mathrm{~d} \nu)$, then $L_{\lambda_{0}}$ is called positive-critical in $\Omega$ with respect to $W$. Otherwise, $L_{\lambda_{0}}$ is called null-critical in $\Omega$ with respect to $W$.

Remark 2.3. Let $\mu \leq \lambda_{0}$, and suppose that $L_{\mu}$ is a subcritical operator in $\Omega$. Let $v$ and $\tilde{v}$ be positive solutions of the equations $L_{\mu} u=0$ and $L_{\mu}^{\star} u=0$ in $\Omega$, respectively, such that $v \tilde{v} \in L^{1}(\Omega, W \mathrm{~d} \nu)$. Since for every fixed $x$ (resp. $y$ ) the function $G_{L_{\mu}}^{\Omega}(x, \cdot)$ (resp. $\left.G_{L_{\mu}}^{\Omega}(\cdot, y)\right)$ is a positive solution of the operator $L_{\mu}^{\star}$ (resp. $L_{\mu}$ ) of minimal growth in $\Omega$, the integrability condition $v \tilde{v} \in L^{1}(\Omega, W \mathrm{~d} \nu)$ implies that

$$
\int_{\Omega} G_{L_{\mu}}^{\Omega}(x, y) W(y) v(y) \mathrm{d} \nu(y)<\infty, \quad \text { and } \int_{\Omega} G_{L_{\mu}}^{\Omega}(x, y) W(x) \tilde{v}(x) \mathrm{d} \nu(x)<\infty .
$$

In light of [27, Lemma 2.1], (2.7) implies that $v$ and $\tilde{v}$ are not $\mu$-invariant positive solutions of the equations $L_{\mu} u=0$ and $L_{\mu}^{\star} u=0$ in $\Omega$, respectively. In other words, if $v \in \mathcal{C}_{L_{\mu}}(\Omega)$ (resp. $\tilde{v} \in \mathcal{C}_{L_{\mu}^{\star}}(\Omega)$ ) is a positive $\mu$-invariant solution of the operator $L$ (resp. $\left.L^{\star}\right)$, and $v \tilde{v} \in L^{1}(\Omega, W \mathrm{~d} \nu)$, then $\mu=\lambda_{0}$ and $L_{\lambda_{0}}$ is positive-critical with respect to $W$ in $\Omega$.

The following example, which is a modification of the counterexamples to Stroock's conjecture given in [27], demonstrates that for $\mu=\lambda_{0}$ there exists a subcritical operator $L_{\lambda_{0}}$ and a potential $W>0$ satisfying all the properties of Remark 2.3 .

Example 2.4. Consider the operator $L:=-\rho \Delta$ on $\mathbb{R}^{d}$, where $d \geq 3$, and $\rho$ is a strictly positive smooth function. Let $W:=\mathbf{1}$. Then $L$ is a subcritical operator in $\mathbb{R}^{d}$, and it follows from Liouville's theorem that the functions $v=\mathbf{1}$ and $\tilde{v}=1 / \rho$ are (up to a multiplicative constant) the unique positive solutions of the equations $L u=0$ and $L^{\star} u=0$ in $\mathbb{R}^{d}$, respectively. We claim that there exists a smooth positive function $\rho$ so that $v \tilde{v}=1 / \rho \in L^{1}\left(\mathbb{R}^{d}, \mathrm{~d} x\right)$, and $\lambda_{0}\left(L, \mathbf{1}, \mathbb{R}^{d}\right)=0$.

Indeed, let $0<\beta<1$, and $x_{k}:=(k, 0, \ldots, 0)$, where, $k=1,2, \ldots$ Finally let $\left\{\varepsilon_{k}\right\} \subset(0,1)$ be a sequence satisfying $\sum_{k=1}^{\infty} \varepsilon_{k}^{d-(2+\beta)}<\infty$. Take a smooth positive function $\tilde{v} \in L^{1}\left(\mathbb{R}^{d}, \mathrm{~d} x\right)$ satisfying $\tilde{v}(x) \uparrow_{B\left(x_{k}, \varepsilon_{k}\right)}=$ $\left(\varepsilon_{k}\right)^{-(2+\beta)}$. In particular, $v \tilde{v} \in L^{1}\left(\mathbb{R}^{d}, \mathbf{1} \mathrm{d} x\right)$. 
On the other hand, we clearly have $\lambda_{0}\left(L, \mathbf{1}, B\left(x_{k}, \varepsilon_{k}\right)\right)<C \varepsilon_{k}^{\beta}$, and therefore, $\lambda_{0}\left(L, \mathbf{1}, \mathbb{R}^{d}\right)=0$. Moreover, by Remark 2.3 , the unique positive solution $v$ (resp. $\tilde{v})$ of the equation $L_{\lambda_{0}} u=0\left(\right.$ resp. $\left.L_{\lambda_{0}}^{\star} u=0\right)$ is not $\lambda_{0^{-}}$ invariant.

Remark 2.5. We note that Example 2.4 is in fact a strengthening of the counterexamples to Stroock's conjecture given in [27]. It gives an example of a subcritical operator $L$ on $\mathbb{R}^{d}, d \geq 3$, with $\lambda_{0}=0$, such that the operators $L$ and $L^{\star}$ do not admit $\lambda_{0}$-invariant positive solutions, and in addition, the product of positive entire solutions of the equations $L u=0$ and $L^{\star} u=0$ is in $L^{1}\left(\mathbb{R}^{d}\right)$. Recall that if a Schrödinger-type operator admits a 'small' positive solution $\psi$ in $\Omega$ (and in particular an $L^{2}$-positive solution), then the operator is critical in $\Omega$, and in particular, $\psi$ is an invariant positive solution [29].

The following notions of small and semismall perturbations play a fundamental role in criticality theory [21, 22, 24, 25]. Semismall perturbations revisit in the present paper. It turns out that they guarantee the compactness of the weighted Green operators in $L^{p}\left(\phi_{p}\right)$ for all $1 \leq p \leq \infty$ (see Section 6).

Definition 2.6. Let $L$ be a subcritical operator in $\Omega$, and let $V$ be a potential.

(i) We say that $V$ is a small perturbation of $L$ in $\Omega$ if

$$
\lim _{k \rightarrow \infty}\left\{\sup _{x, y \in \Omega_{k}^{\star}} \int_{\Omega_{k}^{\star}} \frac{G_{L}^{\Omega}(x, z)|V(z)| G_{L}^{\Omega}(z, y)}{G_{L}^{\Omega}(x, y)} \mathrm{d} \nu(z)\right\}=0 .
$$

(ii) We say that $V$ is a semismall perturbation of $L$ in $\Omega$ if for some (all) fixed $x_{0} \in \Omega$ we have

$$
\lim _{k \rightarrow \infty}\left\{\sup _{y \in \Omega_{k}^{\star}} \int_{\Omega_{k}^{\star}} \frac{G_{L}^{\Omega}\left(x_{0}, z\right)|V(z)| G_{L}^{\Omega}(z, y)}{G_{L}^{\Omega}\left(x_{0}, y\right)} \mathrm{d} \nu(z)\right\}=0 .
$$

Remark 2.7. ( $i$ ) A small perturbation of $L$ in $\Omega$ is a semismall perturbation of $L$ and $L^{\star}$ in $\Omega$ [22].

(ii) We note that $\lambda_{0}$ is well defined by (2.4) even if the potential $W$ does not have a definite sign. It turns out [22, 25] that if $L$ is subcritical and $W \nless 0$ is a semismall (resp. small) perturbation of $L^{\star}$ in $\Omega$, then $\lambda_{0}>0$, and $L_{\lambda_{0}}$ is critical in $\Omega$ with a ground state $\phi$. Moreover, for each $\lambda<\lambda_{0}$ such that the positive Green function $G_{L_{\lambda}}^{\Omega}$ exists there exists a positive constant $C_{\lambda, x_{0}, \varepsilon}$ (resp. $\left.C_{\lambda}\right)$ such that

$$
\begin{aligned}
& \left(C_{\lambda, x_{0}, \varepsilon}\right)^{-1} G_{L_{\lambda}}^{\Omega}\left(x, x_{0}\right) \leq \phi(x) \leq C_{\lambda, x_{0}, \varepsilon} G_{L_{\lambda}}^{\Omega}\left(x, x_{0}\right) \quad \forall x \in \Omega, \operatorname{dist}\left(x, x_{0}\right)>\varepsilon, \\
& \text { (resp. } \left.\left(C_{\lambda}\right)^{-1} G_{L_{\lambda}}^{\Omega}(x, y) \leq G_{L}^{\Omega}(x, y) \leq C_{\lambda} G_{L_{\lambda}}^{\Omega}(x, y) \quad \forall x, y \in \Omega, x \neq y\right) .
\end{aligned}
$$


Since $L_{\lambda_{0}}$ is critical if and only if $L_{\lambda_{0}}^{\star}$ is critical, (2.6) and (2.10) imply that if $W>0$ is a semismall (resp. small) perturbation of $L^{\star}$ in $\Omega$, then $L_{\lambda_{0}}$ is positive-critical. In particular, $\phi$ satisfies (2.6).

(iii) Murata 23] proved that if $L$ is symmetric and the corresponding (Dirichlet) semigroup generated by $L$ is intrinsically ultracontractive on $L^{2}(\Omega)$ [12], then 1 is a small perturbation of $L$ in $\Omega$. On the other hand, an example of Bañuelos and Davis in [5] gives us a finite area domain $\Omega \subset \mathbb{R}^{2}$ such that 1 is a small perturbation of the Laplacian in $\Omega$, but the corresponding semigroup is not intrinsically ultracontractive.

2.2. Functional spaces. Let $B$ be a Banach space and $B^{\star}$ its dual. If $T: B \rightarrow B$ is a (bounded) operator on $B$, we denote by $T^{\star}$ its dual, and the operator norm of $T$ by $\|T\|_{B}$. The range and the kernel of $T$ are denoted by $R(T)$ and $N(T)$, respectively. We denote by $\sigma(T), \sigma_{\text {point }}(T)$ and $\rho(T)$ the spectrum, the point-spectrum, and the resolvent set of the operator $T$. If $\lambda \in \rho(T)$, then we denote by $R(\lambda, T):=(\lambda I-T)^{-1}$ the resolvent of $T$, where $\mathrm{I}$ is the identity map on $B$. For every $f \in B$ and $g^{\star} \in B^{\star}$ we use the notation $\left\langle g^{\star}, f\right\rangle:=g^{\star}(f)$. If $T$ acts on two Banach spaces $X$ and $Y$, we distinguish the operators by using the notation $T \uparrow_{X}, T \uparrow_{Y}$, respectively.

Let $1 \leq p<\infty$, and let $w$ be a fixed (strictly) positive measurable weight function defined on $\Omega$. Denote the real ordered Banach space

$$
L^{p}(w):=L^{p}\left(\Omega, w^{p} \mathrm{~d} \nu\right)=\left\{u \mid u w \in L^{p}(\Omega, \mathrm{d} \nu)\right\}
$$

equipped with the norm

$$
\|u\|_{p, w}:=\|u w\|_{p}=\left[\int_{\Omega}|u(x) w(x)|^{p} \mathrm{~d} \nu(x)\right]^{1 / p} .
$$

For $p=\infty$, let

$$
L^{\infty}(w):=\left\{u \mid u w \in L^{\infty}(\Omega, \mathrm{d} \nu)\right\}
$$

equipped with the norm

$$
\|u\|_{\infty, w}:=\|u w\|_{\infty}=\operatorname{ess} \sup _{\Omega}(|u| w) .
$$

The ordering on $L^{p}(w)$ is the natural pointwise ordering of functions. For the purpose of spectral theory, we consider also the canonical complexification of $L^{p}(w)$ without changing our notation.

For $1 \leq p \leq \infty$, let $p^{\prime}$ be the usual conjugate exponent of $p$, so, $\frac{1}{p}+\frac{1}{p^{\prime}}=1$. It is well-known that for $1 \leq p<\infty,\left(L^{p}(w)\right)^{\star}=L^{p^{\prime}}\left(w^{-1}\right)$, and in particular, the space $L^{p}(w)$ is reflexive for all $1<p<\infty$.

Let $W, \phi, \tilde{\phi}$ be positive continuous functions in $\Omega$. For $1 \leq p \leq \infty$, denote

$$
\phi_{p}:=\phi^{-1}(\phi W \tilde{\phi})^{1 / p}, \quad \tilde{\phi}_{p}:=\tilde{\phi}^{-1}(\phi W \tilde{\phi})^{1 / p},
$$

and consider the corresponding family of weighted Lebesgue spaces $L^{p}\left(\phi_{p}\right)$, and $L^{p}\left(\tilde{\phi}_{p}\right)$. 
We note that $L^{1}\left(\phi_{1}\right)$ is independent of $\phi$ while $L^{\infty}\left(\phi_{\infty}\right)$ is independent of $\tilde{\phi}$ and $W$. Moreover, if $\tilde{\phi}=\phi$ (which is often the case when $L$ is symmetric), then $L^{2}\left(\phi_{2}\right)=L^{2}(\Omega, W \mathrm{~d} \nu)$ and this space is $\phi$ independent.

It can be easily checked that for $1 \leq p<\infty$ we have

$$
\left(L^{p}\left(\phi_{p}\right)\right)^{\star}=L^{p^{\prime}}\left(\tilde{\phi}_{p^{\prime}}\right),
$$

where the pairing between $L^{p}\left(\phi_{p}\right)$ and $L^{p^{\prime}}\left(\tilde{\phi}_{p^{\prime}}\right)$ is given by

$$
<g^{\star}, f>=\int_{\Omega} g^{\star}(x) W(x) f(x) \mathrm{d} \nu(x) \quad \forall g^{\star} \in L^{p^{\prime}}\left(\tilde{\phi}_{p^{\prime}}\right), f \in L^{p}\left(\phi_{p}\right) .
$$

Here the duality is provided by the bilinear rather than the sesquilinear form 1 .

Suppose now that

$$
\phi W \tilde{\phi} \in L^{1}(\Omega, \mathrm{d} \nu), \quad \int_{\Omega} \phi(x) W(x) \tilde{\phi}(x) \mathrm{d} \nu(x)=1 .
$$

Then by the Hölder inequality we have the continuous embeddings

$$
L^{\infty}\left(\phi_{\infty}\right) \subset L^{q}\left(\phi_{q}\right) \subset L^{p}\left(\phi_{p}\right) \subset L^{1}\left(\phi_{1}\right),
$$

for all $1 \leq p \leq q \leq \infty$, and for $f \in L^{\infty}\left(\phi_{\infty}\right)$ we have

$$
\|f\|_{1, W \tilde{\phi}}=\|f\|_{1, \phi_{1}} \leq\|f\|_{p, \phi_{p}} \leq\|f\|_{q, \phi_{q}} \leq\|f\|_{\infty, \phi_{\infty}}=\|f\|_{\infty, \phi^{-1}} .
$$

Moreover, $\|f\|_{1, \phi_{1}}=\|f\|_{\infty, \phi_{\infty}}=1$ if and only if $|f|=\phi=1$ almost everywhere. In particular, $\phi \in L^{p}\left(\phi_{p}\right)$ for every $1 \leq p \leq \infty$, and (2.13) implies that

$$
\|\phi\|_{1, \phi_{1}}=\|\phi\|_{p, \phi_{p}}=\|\phi\|_{\infty, \phi_{\infty}}=1 \quad \forall 1 \leq p \leq \infty,
$$

so, the norms of the embeddings in (2.14) is 1 . Moreover, these embeddings are dense. We also note that if $\tilde{\phi}=\phi$ (as in the symmetric case), then

$$
\left(L^{1}(W \phi)\right)^{\star}=L^{\infty}\left(\phi^{-1}\right) \subset L^{2}(\Omega, W \mathrm{~d} \nu) \subset L^{1}(W \phi) .
$$

Remark 2.8. Throughout the paper we fix an operator $L$ of the form (2.1), a positive potential $W, \mu \leq \lambda_{0}$, and $\phi, \tilde{\phi}$ two positive solutions of the equations $L_{\mu} u=0$ and $L_{\mu}^{\star} u=0$ in $\Omega$, respectively. We study properties of a family of the corresponding weighted Green operators on $L^{p}\left(\phi_{p}\right)$ and $L^{p}\left(\tilde{\phi}_{p}\right)$. We note that if $\mu=\lambda_{0}$ and $L_{\lambda_{0}}$ is critical in $\Omega$, then the spaces $L^{p}\left(\phi_{p}\right)$ and $L^{p}\left(\tilde{\phi}_{p}\right)$ are uniquely defined.

Remark 2.9. Let $\phi$ and $\tilde{\phi}$ be two fixed positive solutions of the equations $L u=0$ and $L^{\star} u=0$ in $\Omega$, respectively. For $\mu \leq \lambda_{0}$, define the operator

$$
L_{\mu}^{\phi}:=\frac{1}{\phi} L_{\mu} \phi=\frac{1}{\phi} L \phi-\mu W=L^{\phi}-\mu W,
$$

\footnotetext{
${ }^{1}$ This is not essential, but simplifies somewhat the calculations.
} 
which is called Doob's $\phi$-transform (or the ground state transform with respect to $\phi$ ) of the operator $L_{\mu}$. Note that for $\mu \leq \lambda_{0}$ the operator $L_{\mu}$ is subcritical in $\Omega$ if and only if $L_{\mu}^{\phi}$ is subcritical in $\Omega$, and we have

$$
G_{L_{\mu}^{\phi}}^{\Omega}(x, y)=\frac{1}{\phi(x)} G_{L_{\mu}}^{\Omega}(x, y) \phi(y) .
$$

Clearly, $L^{\phi} \mathbf{1}=0$ and $\left(L^{\phi}\right)^{\star}(\phi \tilde{\phi})=0$. In particular, $L^{\phi}$ is a diffusion operator. We note that for $1 \leq p \leq \infty$, the weighted $L^{p}$-spaces associated with the positive solutions 1 and $\phi \tilde{\phi}$ of the equations $L^{\phi} u=0$ and $\left(L^{\phi}\right)^{\star} u=0$, respectively, are just $L^{p}(\Omega, \phi W \tilde{\phi} \mathrm{d} \nu)$. So, in this case (which corresponds to the class of diffusion operators) the corresponding one-parameter weights are $p$-independent.

\section{Boundedness of the Green operators}

Fix a positive potential $W$ and $\mu \leq \lambda_{0}$. Let $\phi$ and $\tilde{\phi}$ be two fixed positive solutions of the equations $L_{\mu} u=0$ and $L_{\mu}^{\star} u=0$ in $\Omega$, respectively. For $1 \leq p \leq \infty$ let $\phi_{p}$ and $\tilde{\phi}_{p}$ be the functions defined in (2.11). Note that we do not assume below neither that $\phi$ and $\tilde{\phi}$ are invariant solutions nor that the integrability condition (2.13) is satisfied.

For $\lambda<\mu$, we introduce the integral operators

$\mathcal{G}_{\lambda} f(x):=\int_{\Omega} G_{L_{\lambda}}^{\Omega}(x, y) W(y) f(y) \mathrm{d} \nu(y), \quad \mathcal{G}_{\lambda}^{\odot} f(y):=\int_{\Omega} G_{L_{\lambda}}^{\Omega}(x, y) W(x) f(x) \mathrm{d} \nu(x)$.

In the present section we study for $1 \leq p \leq \infty$ the boundedness of the weighted Green operators $\mathcal{G}_{\lambda}$ and $\mathcal{G}_{\lambda}^{\odot}$ on $L^{p}\left(\phi_{p}\right)$ and $L^{p}\left(\tilde{\phi}_{p}\right)$, respectively. We have

Theorem 3.1. Let $L$ be an elliptic operator on $\Omega$ of the form (2.1), and let $W$ be a positive potential. Fix $\mu \leq \lambda_{0}$, and let $\phi$ and $\tilde{\phi}$ be two fixed positive solutions of the equations $L_{\mu} u=0$ and $L_{\mu}^{\star} u=0$ in $\Omega$, respectively. Then

(1) For $1 \leq p \leq \infty$, the operator $\mathcal{G}_{\lambda} \uparrow_{L^{p}\left(\phi_{p}\right)}$ (resp., $\mathcal{G}_{\lambda}^{\odot} \uparrow_{L^{p}\left(\tilde{\phi}_{p}\right)}$ ) is a well defined bounded and positive improving operator on $L^{p}\left(\phi_{p}\right)$ (resp. $\left.L^{p}\left(\tilde{\phi}_{p}\right)\right)$. Moreover, we have

$$
\left.\left\|\mathcal{G}_{\lambda}\right\|_{L^{p}\left(\phi_{p}\right)} \leq(\mu-\lambda)^{-1}, \quad \text { (resp. }\left\|\mathcal{G}_{\lambda}^{\odot}\right\|_{L^{p}\left(\tilde{\phi}_{p}\right)} \leq(\mu-\lambda)^{-1}\right) .
$$

(2) For $1 \leq p<\infty$, the operator $\mathcal{G}_{\lambda}^{\odot} \uparrow_{L^{p^{\prime}}\left(\tilde{\phi}_{p^{\prime}}\right)}$ is the dual operator of $\mathcal{G}_{\lambda} \uparrow_{L^{p}\left(\phi_{p}\right)}$, and $\mathcal{G}_{\lambda} \uparrow_{L^{p^{\prime}}\left(\phi_{p^{\prime}}\right)}$ is the dual of $\mathcal{G}_{\lambda}^{\odot} \uparrow_{L^{p}\left(\tilde{\phi}_{p}\right)}$.

(3) Suppose that $\phi$ is a $\mu$-invariant positive solution of the operator $L$, and $p=\infty$, then $\left\|\mathcal{G}_{\lambda}\right\|_{L^{\infty}\left(\phi_{\infty}\right)}=(\mu-\lambda)^{-1}$.

(4) Suppose that $\phi$ is a $\mu$-invariant positive solution of the operator $L$ satisfying (2.13), then for any $1 \leq p \leq \infty,\left\|\mathcal{G}_{\lambda}\right\|_{L^{p}\left(\phi_{p}\right)}=(\mu-\lambda)^{-1}$. 
Proof. (1) Let $f \in L^{\infty}\left(\phi_{\infty}\right)$, then by (2.5)

$$
\begin{aligned}
\left|\mathcal{G}_{\lambda} f(x)\right| \leq & \int_{\Omega} G_{L_{\lambda}}^{\Omega}(x, y) W(y)|f(y)| \mathrm{d} \nu(y) \leq \\
& \|f\|_{\infty, \phi_{\infty}} \int_{\Omega} G_{L_{\lambda}}^{\Omega}(x, y) W(y) \phi(y) \mathrm{d} \nu(y) \leq \frac{\|f\|_{\infty, \phi_{\infty}}}{\mu-\lambda} \phi(x),
\end{aligned}
$$

so, $\left\|\mathcal{G}_{\lambda}\right\|_{L^{\infty}\left(\phi_{\infty}\right)} \leq(\mu-\lambda)^{-1}$. Similarly, $\left\|\mathcal{G}_{\lambda}^{\odot}\right\|_{L^{\infty}\left(\tilde{\phi}_{\infty}\right)} \leq(\mu-\lambda)^{-1}$.

Assume now that $f \in L^{1}\left(\phi_{1}\right)$, then by the Tonelli-Fubini theorem and (2.5) we obtain

$$
\begin{gathered}
\left\|\mathcal{G}_{\lambda} f(x)\right\|_{1, \phi_{1}}=\int_{\Omega} W(x) \tilde{\phi}(x)\left|\int_{\Omega} G_{L_{\lambda}}^{\Omega}(x, y) W(y) f(y) \mathrm{d} \nu(y)\right| \mathrm{d} \nu(x) \leq \\
\int_{\Omega} W(x) \tilde{\phi}(x) \int_{\Omega} G_{L_{\lambda}}^{\Omega}(x, y) W(y)|f(y)| \mathrm{d} \nu(y) \mathrm{d} \nu(x)= \\
\int_{\Omega}\left(\int_{\Omega} W(x) \tilde{\phi}(x) G_{L_{\lambda}}^{\Omega}(x, y) \mathrm{d} \nu(x)\right) W(y)|f(y)| \mathrm{d} \nu(y) \leq \\
\frac{1}{\mu-\lambda} \int_{\Omega} \tilde{\phi}(y) W(y)|f(y)| \mathrm{d} \nu(y)=\frac{\|f\|_{1, \phi_{1}}}{\mu-\lambda} .
\end{gathered}
$$

Hence, $\left\|\mathcal{G}_{\lambda}\right\|_{L^{1}\left(\phi_{1}\right)} \leq(\mu-\lambda)^{-1}$. Similarly, $\left\|\mathcal{G}_{\lambda}^{\odot}\right\|_{L^{1}\left(\tilde{\phi}_{1}\right)} \leq(\mu-\lambda)^{-1}$.

For $1<p<\infty$, the boundedness of $\mathcal{G}_{\lambda} \Gamma_{L^{p}\left(\phi_{p}\right)}$ with norm estimate $\left\|\mathcal{G}_{\lambda}\right\|_{L^{p}\left(\phi_{p}\right)} \leq(\mu-\lambda)^{-1}$ follows now directly from a Riesz-Thorin-type interpolation theorem with weights proved by Stein [35, Theorem 2].

(2) The duality claim follows now directly from (2.12).

(3) and (4) follow from (1) and (2.6).

Remark 3.2. Theorem 3.1 (and (4.2)) for $1<p<\infty$ follows also from the Schur test with weights [14, Lemma 5.1]. Indeed, set

$$
K(x, y):=\frac{G(x, y)}{\phi^{1-p}(y) \tilde{\phi}(y)}, \quad w(x, y):=\frac{\phi^{p}(y)}{\phi^{p}(x)}, \quad \mathrm{d} \rho(y):=\left(\phi_{p}(y)\right)^{p} \mathrm{~d} \nu(y) .
$$

Then (2.5) implies that

$$
\begin{gathered}
\int_{\Omega} w(x, y)^{\frac{1}{p}} K(x, y) \mathrm{d} \rho(y)=\frac{\int_{\Omega} G_{L_{\lambda}}^{\Omega}(x, y) W(y) \phi(y) \mathrm{d} \nu(y)}{\phi(x)} \leq \frac{1}{\mu-\lambda} \quad \forall x \in \Omega, \\
\int_{\Omega} w(x, y)^{-\frac{1}{p^{\prime}}} K(x, y) \mathrm{d} \rho(x)=\frac{\int_{\Omega} G_{L_{\lambda}}^{\Omega}(x, y) W(x) \tilde{\phi}(x) \mathrm{d} \nu(x)}{\tilde{\phi}(y)} \leq \frac{1}{\mu-\lambda} \quad \forall y \in \Omega .
\end{gathered}
$$

Applying the aforementioned Schur test we get $\left\|\mathcal{G}_{\lambda}\right\|_{L^{p}\left(\phi_{p}\right)} \leq(\mu-\lambda)^{-1}$. 
The Schur test with weights is essentially a theorem of Aronszajn, and in fact follows from Stein's Riesz-Thorin-type interpolation theorem with weights [35, Theorem 2].

Remark 3.3. It follows from part (ii) of Theorem 4.4 that the assumptions of part (4) of Theorem 3.1 imply that in fact $\mu=\lambda_{0}$ and $L_{\lambda_{0}}$ is positivecritical in $\Omega$ with respect to $W$.

Remark 3.4. The norm estimate (3.1) does not depend on $\phi, \tilde{\phi}$ and $W$ and $p$.

Remark 3.5. The requirement that $W$ is strictly positive can be weakened, and Theorem 3.1 holds in a slightly weaker sense if $W$ is a nonzero nonnegative function. Indeed, let $1 \leq p \leq \infty$. Since Stein's Riesz-Thorin-type interpolation theorem with weights [35, Theorem 2] holds for nonnegative weights, we have for $\lambda<\mu$

$$
\left\|\left(\mathcal{G}_{\lambda} f\right) \phi_{p}\right\|_{L^{p}(\Omega, \mathrm{d} \nu)} \leq \frac{1}{\mu-\lambda}\left\|f \phi_{p}\right\|_{L^{p}(\Omega, \mathrm{d} \nu)} \quad \forall f \text { s.t. } f \phi_{p} \in L^{p}(\Omega, \mathrm{d} \nu) .
$$

\section{Principal eigenfunction}

The Krein-Rutman theorem roughly asserts that if $T$ is a compact operator defined on a Banach space $X$ with a total cone $P$ such that $T$ is positive improving and its spectral radius $r(T)$ is strictly positive, then $T$ admits a positive eigenfunction with an eigenvalue $r(T)$. Moreover, under an irreducibility assumption $r(T)$ is simple. The weighted Green operator $\mathcal{G}_{\lambda}$ in the weighted Lebesgue spaces $L^{p}\left(\phi_{p}\right)$ is positive improving but in general, $\mathcal{G}_{\lambda}$ is not compact. Nevertheless, under some further conditions it admits a positive eigenfunction with an eigenvalue $(\mu-\lambda)^{-1}$.

Throughout the present section, as in Section 3, $W$ is a fixed positive potential, $\mu \leq \lambda_{0}$, and $\phi, \tilde{\phi}$ are fixed positive solutions of the equations $L_{\mu} u=0$ and $L_{\mu}^{\star} u=0$ in $\Omega$, respectively. We study eigenvalues and eigenfunctions of the weighted Green operators $\mathcal{G}_{\lambda}$.

Remark 4.1. Eigenfunctions of $\mathcal{G}_{\lambda} \uparrow_{L^{1}\left(\phi_{1}\right)}$ might be not smooth enough to solve weakly the corresponding partial differential equation. Therefore, if $p=1$, we always assume that such eigenfunctions are also in $L_{\text {loc }}^{q}(\Omega)$ for some $q>1$.

We have

Theorem 4.2. Let $W, \mu, \phi$, and $\tilde{\phi}$ be as above, and let $\lambda<\mu$. Then for any $1 \leq p \leq \infty$, zero is not an eigenvalue of the operators $\mathcal{G}_{\lambda} \uparrow_{L^{p}}\left(\phi_{p}\right)$ and $\mathcal{G}_{\lambda}^{\odot} \uparrow_{L^{p}}\left(\tilde{\phi}_{p}\right) \cdot$

Moreover, any eigenfunction $\varphi$ (resp. $\tilde{\varphi})$ of $\mathcal{G}_{\lambda} \uparrow_{L^{p}\left(\phi_{p}\right)}$ (resp. $\left.\mathcal{G}_{\lambda}^{\odot} \uparrow_{L^{p}\left(\tilde{\phi}_{p}\right)}\right)$ with an eigenvalue $\nu$ solves the equation

$$
\left(L-\left[\lambda+(\nu)^{-1}\right] W\right) \varphi=0 \quad\left(\operatorname{resp} .\left(L^{\star}-\left[\lambda+(\nu)^{-1}\right] W\right) \tilde{\varphi}=0\right) \quad \text { in } \Omega .
$$


Proof. Let $1 \leq p \leq \infty$ and let $\varphi \in L^{p}\left(\phi_{p}\right),\|\varphi\|_{p, \phi_{p}}=1$ be an eigenfunction of the operator $\mathcal{G}_{\lambda} \uparrow_{L^{p}\left(\phi_{p}\right)}$ with an eigenvalue $\nu$, and define

$$
\varphi_{k}(x):=\int_{\Omega_{k}} G_{L_{\lambda}}^{\Omega_{k}}(x, y) W(y) \varphi(y) \mathrm{d} \nu(y) \quad x \in \Omega_{k}, k \geq 1 .
$$

Clearly,

$$
G_{L_{\lambda}}^{\Omega_{k}}(x, y) W(y)|\varphi(y)| \leq G_{L_{\lambda}}^{\Omega}(x, y) W(y)|\varphi(y)| \quad \text { in } \Omega_{k} .
$$

On the other hand, by Theorem 3.1 we have $G_{L_{\lambda}}^{\Omega}(x, \cdot) W \varphi \in L^{1}(\Omega, \mathrm{d} \nu)$ for almost every $x \in \Omega$. Therefore, $\varphi_{k}(x)$ is well-defined almost everywhere in $\Omega_{k}$, and Lebesgue's dominated convergence theorem implies that

$$
\lim _{k \rightarrow \infty} \varphi_{k}(x)=\int_{\Omega} G_{L_{\lambda}}^{\Omega}(x, y) W(y) \varphi(y) \mathrm{d} \nu(y)=\nu \varphi(x)
$$

almost everywhere in $\Omega$.

Since $|\varphi| \in L^{p}\left(\phi_{p}\right)$, Theorem 3.1 implies that $\mathcal{G}_{\lambda}|\varphi| \in L^{p}\left(\phi_{p}\right)$. Obviously, $\left|\varphi_{k}\right| \leq \mathcal{G}_{\lambda}|\varphi| \in L^{p}\left(\phi_{p}\right)$. Consequently, $\left\{\varphi_{k}\right\}$ is bounded in $L^{p}\left(\phi_{p}\right)$. Note that for $1 \leq p<\infty$, Lebesgue's dominated convergence theorem implies that $\left\|\varphi_{k}\right\|_{L^{p}\left(\phi_{p}\right)} \rightarrow|\nu|\|\varphi\|_{L^{p}\left(\phi_{p}\right)}$, and this holds true also for $p=\infty$.

On the other hand, taking into account Remark 4.1 in case $p=1$, it follows that each $\varphi_{k}$ solves the equation

$$
(L-\lambda W) \varphi_{k}=W \varphi \quad \text { in } \Omega_{k} .
$$

A standard elliptic regularity argument implies that $\nu \varphi$ solves the equation

$$
(L-\lambda W) \nu \varphi=W \varphi \neq 0 \quad \text { in } \Omega .
$$

In particular, $\nu \neq 0$. Thus, $\varphi$ solves the equation

$$
\left(L-\left[\lambda+(\nu)^{-1}\right] W\right) \varphi=0 \quad \text { in } \Omega .
$$

Remark 4.3. It was proved in [28] that zero is not an eigenvalue of $\mathcal{G}_{\lambda}\left\lceil_{C\left(\phi_{\infty}\right)}\right.$.

The next result concerns conditions under which the positive solution $\phi$ is an eigenfunction of $\mathcal{G}_{\lambda}$ in $L^{p}\left(\phi_{p}\right)$.

Theorem 4.4. Let $W, \mu, \phi$, and $\tilde{\phi}$ be as above, and let $\lambda<\mu$. Then

(i) The function $\phi$ (resp. $\tilde{\phi})$ is a nonnegative eigenfunction of the operator $\mathcal{G}_{\lambda}\left\lceil_{L^{\infty}\left(\phi_{\infty}\right)}\right.$ (resp., $\left.\mathcal{G}_{\lambda}^{\odot} \uparrow_{L^{\infty}\left(\tilde{\phi}_{\infty}\right)}\right)$ with an eigenvalue $(\mu-\lambda)^{-1}$ if and only if $\phi$ (resp. $\tilde{\phi})$ is a $\mu$-invariant positive solution with respect to $L$ (resp. $\left.L^{\star}\right)$. In this case

$$
\begin{aligned}
\left\|\mathcal{G}_{\lambda}\right\|_{L^{\infty}\left(\phi_{\infty}\right)}=\left\|\mathcal{G}_{\lambda}^{\odot}\right\|_{L^{1}\left(\tilde{\phi}_{1}\right)}=(\mu-\lambda)^{-1} & \\
& \left(\text { resp. }\left\|\mathcal{G}_{\lambda}^{\odot}\right\|_{L^{\infty}\left(\tilde{\phi}_{\infty}\right)}=\left\|\mathcal{G}_{\lambda}\right\|_{L^{1}\left(\phi_{1}\right)}=(\mu-\lambda)^{-1}\right) .
\end{aligned}
$$


Furthermore, if $\phi$ and $\tilde{\phi}$ are both $\mu$-invariant positive solutions, then for $1 \leq p \leq \infty$

$$
\left\|\mathcal{G}_{\lambda}\right\|_{L^{p}\left(\phi_{p}\right)}=\left\|\mathcal{G}_{\lambda}^{\odot}\right\|_{L^{p}\left(\tilde{\phi}_{p}\right)}=(\mu-\lambda)^{-1}
$$

(ii) Let $1 \leq p<\infty$. Then $\phi$ (resp. $\tilde{\phi}$ ) is a nonnegative eigenfunction of the operator $\left.\mathcal{G}_{\lambda}\right\rceil_{L^{p}\left(\phi_{p}\right)}$ (resp., $\mathcal{G}_{\lambda}^{\odot} \uparrow_{L^{p}\left(\tilde{\phi}_{p}\right)}$ ) with an eigenvalue $\nu=(\mu-\lambda)^{-1}$ if and only if $\mu=\lambda_{0}$, and the operator $L_{\lambda_{0}}$ is positive-critical with respect to $W$. In this case, $\phi$ and $\tilde{\phi}$ are the ground states of $L_{\lambda_{0}}$ and $L_{\lambda_{0}}^{\star}$, respectively, and

$$
\left\|\mathcal{G}_{\lambda}\right\|_{L^{p}\left(\phi_{p}\right)}=\left\|\mathcal{G}_{\lambda}^{\odot}\right\|_{L^{p}\left(\tilde{\phi}_{p}\right)}=\frac{1}{\lambda_{0}-\lambda} \quad \forall 1 \leq p \leq \infty .
$$

Proof. (i) All the claims of this part can be checked easily and left to the reader. In particular, use Theorem 3.1 and Stein's Riesz-Thorin-type interpolation theorem to prove (4.2).

(ii) Let $1 \leq p<\infty$. The positive solution $\phi$ is an eigenfunction of the operator $\mathcal{G}_{\lambda}\left\lceil_{L^{p}\left(\phi_{p}\right)}\right.$ with an eigenvalue $(\mu-\lambda)^{-1}$ if and only if $\phi W \tilde{\phi} \in L^{1}(\Omega)$ and $\phi$ is $\mu$-invariant positive solution.

In particular, if $\mu=\lambda_{0}$, and $L_{\lambda_{0}}$ is positive-critical with respect to $W$, then $\phi$ is an eigenfunction of the operator $\mathcal{G}_{\lambda} \Gamma_{L^{p}\left(\phi_{p}\right)}$ with an eigenvalue $\left(\lambda_{0}-\lambda\right)^{-1}$.

On the other hand, if $\phi$ is an eigenfunction of the operator $\left.\mathcal{G}_{\lambda}\right|_{L^{p}}\left(\phi_{p}\right)$ with an eigenvalue $(\mu-\lambda)^{-1}$, then $\phi W \tilde{\phi} \in L^{1}(\Omega)$.

Assume that $L_{\mu}$ is subcritical in $\Omega$, then

$$
\int_{\Omega} G_{L_{\mu}}^{\Omega}(x, y) W(y) \phi(y) \mathrm{d} \nu(y)<\infty .
$$

By Remark 2.3, $\phi$ is not a $\mu$-invariant solution, and we get a contradiction. Therefore, $L_{\mu}$ is critical in $\Omega$ and hence, $\mu=\lambda_{0}$. Since $\phi W \tilde{\phi} \in L^{1}(\Omega)$, it follows that $L_{\lambda_{0}}$ is positive-critical with respect to $W$.

In the critical case we have the following result.

Theorem 4.5. Let $L$ be an elliptic operator on $\Omega$ of the form (2.1), and let $W$ be a positive potential. Assume that the operator $L_{\lambda_{0}}$ is critical, and let $\phi$ and $\tilde{\phi}$ be the ground states of $L_{\lambda_{0}}$ and $L_{\lambda_{0}}^{\star}$, respectively. Fix $\lambda<\lambda_{0}$. Then

(i) For $1 \leq p \leq \infty$, we have

$$
\left\|\mathcal{G}_{\lambda}\right\|_{L^{p}\left(\phi_{p}\right)}=\left\|\mathcal{G}_{\lambda}^{\odot}\right\|_{L^{p}\left(\tilde{\phi}_{p}\right)}=\frac{1}{\lambda_{0}-\lambda} .
$$

(ii) The operator $\mathcal{G}_{\lambda} \uparrow_{L^{\infty}\left(\phi_{\infty}\right)}$ (resp., $\mathcal{G}_{\lambda}^{\odot} \uparrow_{L^{\infty}\left(\tilde{\phi}_{\infty}\right)}$ ) admits a unique eigenvalue $\nu=\left(\lambda_{0}-\lambda\right)^{-1}$ with a nonnegative eigenfunction. Moreover, $\left(\lambda_{0}-\lambda\right)^{-1}$ is a simple eigenvalue of $\left.\mathcal{G}_{\lambda}\right|_{L^{\infty}\left(\phi_{\infty}\right)}$ (resp., $\left.\mathcal{G}_{\lambda}^{\odot} \uparrow_{L^{\infty}\left(\tilde{\phi}_{\infty}\right)}\right)$. The corresponding 
eigenfunction is $\phi$ (resp., $\tilde{\phi})$, and $\phi$ (resp. $\tilde{\phi})$ is the unique $L^{\infty}\left(\phi_{\infty}\right)$ (resp. $\left.L^{\infty}\left(\tilde{\phi}_{\infty}\right)\right)$ solution of the equation $L_{\lambda_{0}} u=0$ in $\Omega$.

(iii) Suppose further that the operator $L_{\lambda_{0}}$ is positive-critical with respect to $W$. Then for all $1 \leq p<\infty$ the function $\phi$ (resp. $\tilde{\phi}$ ) is the unique (up to a multiplicative constant) nonnegative eigenfunction of the operator $\left.\mathcal{G}_{\lambda}\right\rceil_{L^{p}}\left(\phi_{p}\right)$ (resp., $\mathcal{G}_{\lambda}^{\odot} \uparrow_{L^{p}}\left(\tilde{\phi}_{p}\right)$ ).

Proof. $(i-i i)$ Since $\phi$ is a ground state, it is a $\lambda_{0}$-invariant positive solution with respect to the operator $L$ and the weight $W$, part $(i)$ and the existence assertion of (ii) follow from part (i) of Theorem 4.4.

It remains to prove the uniqueness and simplicity of $\left(\lambda_{0}-\lambda\right)^{-1}$ for the operator $\left.\mathcal{G}_{\lambda}\right|_{L^{\infty}\left(\phi_{\infty}\right)}$. Let $\varphi$ be a nonnegative eigenfunction of the operator $\mathcal{G}_{\lambda} \Gamma_{L^{\infty}\left(\phi_{\infty}\right)}$ with an eigenvalue $\nu=(\kappa-\lambda)^{-1}$. Without loss of generality we may assume that $\|\varphi\|_{\infty, \phi_{\infty}}=1$, thus $\phi-\varphi \geq 0$. By Theorem 4.2, $\varphi$ is a positive solution of the equation $(L-\kappa W) u=0$ in $\Omega$. Hence, $\kappa \leq$ $\lambda_{0}$. Therefore, $v:=\phi-\varphi$ is a nonnegative supersolution of the equation $\left(L-\lambda_{0} W\right) u=$ in $\Omega$. On the other hand, $L_{\lambda_{0}}$ is critical in $\Omega$ if and only if $\phi$ is the unique (up to a multiplicative constant) nonzero nonnegative supersolution. Thus, $\kappa=\lambda_{0}$ and $\varphi=\phi$. Hence, for all $\lambda<\lambda_{0}$, $\phi$ is the unique nonnegative eigenfunction of $\left.\mathcal{G}_{\lambda}\right\rceil_{L^{\infty}}\left(\phi_{\infty}\right)$.

Moreover, if $\left(\lambda_{0}-\lambda\right)^{-1}$ is an eigenvalue of $\left.\mathcal{G}_{\lambda}\right|_{L^{\infty}\left(\phi_{\infty}\right)}$ with a normalized eigenfunction $\varphi$, then $u:=\phi-\varphi \geq 0$, and by Theorem $4.2 u$ is a nonnegative solution of the equation $L_{\lambda_{0}} u=0$ in $\Omega$. Since $L_{\lambda_{0}}$ is critical, it follows that $u=c \phi$ for some $c \geq 0$. So, $\varphi=(1-c) \phi$, and either $c=0$ or $c=2$. Hence, $\left(\lambda_{0}-\lambda\right)^{-1}$ is a simple eigenvalue of $\mathcal{G}_{\lambda}\left\lceil_{L^{\infty}}\left(\phi_{\infty}\right)\right.$.

(iii) For $1 \leq p<\infty$, part (ii) of Theorem 4.4 implies that $\phi$ is a positive eigenfunction of $\mathcal{G}_{\lambda}\left\lceil_{L^{p}}\left(\phi_{p}\right)\right.$ with an eigenvalue $\left(\lambda_{0}-\lambda\right)^{-1}$.

Let $\varphi \in L^{p}\left(\phi_{p}\right)$ be a nonnegative normalized eigenfunction of the operator $\left.\mathcal{G}_{\lambda}\right\rceil_{L^{p}\left(\phi_{p}\right)}$ with an eigenvalue $\nu$. Note that $\varphi$ is strictly positive since $\mathcal{G}_{\lambda}\left\lceil_{L^{p}}\left(\phi_{p}\right)\right.$ is positivity improving. Clearly, $\nu \geq 0$, and by Theorem $4.2 \nu \neq 0$. Therefore, $\nu$ can be written as $\nu=(\kappa-\lambda)^{-1}$, where $\lambda<\kappa$.

On the other hand, $\nu \leq\left\|\mathcal{G}_{\lambda}\right\|_{L^{p}\left(\phi_{p}\right)}=\left(\lambda_{0}-\lambda\right)^{-1}$, and hence $\lambda_{0} \leq \kappa$. By Theorem 4.2, $\varphi$ is a nonnegative solution of the equation $L_{\kappa} u=0$ in $\Omega$, therefore $\kappa \leq \lambda_{0}$, Thus, $\kappa=\lambda_{0}$, and $\varphi=\phi$.

The next result deals with the case $p=2$. In this case, any $L^{2}$-eigenfunction of $\mathcal{G}_{\lambda} \uparrow_{L^{2}\left(\phi_{2}\right)}$ with the 'maximal' eigenvalue has a definite sign and this eigenvalue is simple.

Theorem 4.6. Let $L$ be an elliptic operator on $\Omega$ of the form (2.1), and let $W$ be a positive potential. Fix $\lambda<\mu \leq \lambda_{0}$, and let $\phi$ and $\tilde{\phi}$ be two positive solutions of the equation $L_{\mu} u=0$ and $L_{\mu}^{\star} u=0$ in $\Omega$, respectively.

If $\varphi$ is an eigenfunction of $\mathcal{G}_{\lambda} \Gamma_{L^{2}\left(\phi_{2}\right)}$ with an eigenvalue $(\mu-\lambda)^{-1}$, then $\varphi$ has a definite sign, and $(\mu-\lambda)^{-1}$ is a simple eigenvalue of $\mathcal{G}_{\lambda} \uparrow_{L^{2}\left(\phi_{2}\right)}$. 
Assume further that $\phi W \tilde{\phi} \in L^{1}(\Omega)$. Then $\varphi=c \phi$ for some constant $c$, $\mu=\lambda_{0}$, and the operator $L_{\lambda_{0}}$ is positive-critical. Moreover, $\left(\lambda_{0}-\lambda\right)^{-1}$ is the unique eigenvalue of $\left.\mathcal{G}_{\lambda}\right|_{L^{p}}\left(\phi_{p}\right)$ with a nonnegative eigenfunction for all $1 \leq p \leq \infty$ and all $\lambda<\lambda_{0}$. Furthermore, $\left(\lambda_{0}-\lambda\right)^{-1}$ is a simple eigenvalue of $\left.\mathcal{G}_{\lambda}\right|_{L^{p}\left(\phi_{p}\right)}$ for all $2 \leq p \leq \infty$, and all $\lambda<\lambda_{0}$.

Proof. Recall that

$$
\left\|\mathcal{G}_{\lambda}\right\|_{L^{2}\left(\phi_{2}\right)} \leq \frac{1}{\mu-\lambda} .
$$

Let $\varphi \in L^{2}\left(\phi_{2}\right)$ be an eigenfunction of the operator $\mathcal{G}_{\lambda} \uparrow_{L^{2}\left(\phi_{2}\right)}$ with an eigenvalue $(\mu-\lambda)^{-1}$. Thus, $\left\|\mathcal{G}_{\lambda}\right\|_{L^{2}\left(\phi_{2}\right)}=(\mu-\lambda)^{-1}$. Without loss of generality, we may assume that $\varphi$ is a real function. Therefore, due to the positivity improving of $\mathcal{G}_{\lambda} \uparrow_{L^{2}\left(\phi_{2}\right)}$, and the Cauchy-Schwarz inequality, we obtain

$$
\begin{gathered}
\frac{1}{\mu-\lambda}\|\varphi\|_{L^{2}\left(\phi_{2}\right)}^{2}=\left(\varphi,\left.\mathcal{G}_{\lambda}\right|_{L^{2}\left(\phi_{2}\right)} \varphi\right)_{L^{2}\left(\phi_{2}\right)} \leq \\
\left(|\varphi|,\left|\mathcal{G}_{\lambda}\right|_{L^{2}\left(\phi_{2}\right)} \varphi \mid\right)_{L^{2}\left(\phi_{2}\right)} \leq\left(|\varphi|,\left.\mathcal{G}_{\lambda}\right|_{L^{2}\left(\phi_{2}\right)}|\varphi|\right)_{L^{2}\left(\phi_{2}\right)} \leq \\
\|\varphi\|_{L^{2}\left(\phi_{2}\right)}\left\|\mathcal{G}_{\lambda} \uparrow_{L^{2}\left(\phi_{2}\right)}|\varphi|\right\|_{L^{2}\left(\phi_{2}\right)} \leq \frac{1}{\mu-\lambda}\|\varphi\|_{L^{2}\left(\phi_{2}\right)}^{2} .
\end{gathered}
$$

As a result we have equality signs in all the inequalities of (4.5). The equality in the Cauchy-Schwarz inequality implies that

$$
\left.\mathcal{G}_{\lambda}\right|_{L^{2}\left(\phi_{2}\right)}|\varphi|=\frac{1}{\mu-\lambda}|\varphi|
$$

and therefore, $|\varphi|$ is a nonnegative eigenfunction of the operator $\mathcal{G}_{\lambda}\left\lceil_{L^{2}}\left(\phi_{2}\right)\right.$. Since $\left.\mathcal{G}_{\lambda}\right|_{L^{2}\left(\phi_{2}\right)}$ is positivity improving, we have $|\varphi|>0$. It follows that any such eigenfunction has a definite sign. Consequently, a standard orthogonality argument shows that $(\mu-\lambda)^{-1}$ is simple (cf. [30, Theorem XIII.43]).

Assume further that $\phi W \tilde{\phi} \in L^{1}(\Omega)$, and denote $\hat{\phi}_{p}:=|\varphi|^{-1}(|\varphi| W \tilde{\phi})^{1 / p}$. Then by the Cauchy-Schwarz inequality $\varphi W \tilde{\phi} \in L^{1}(\Omega)$, and hence $|\varphi|$ is a positive eigenfunction of the operator $\mathcal{G}_{\lambda} \uparrow_{L^{2}\left(\hat{\phi}_{2}\right)}$. Therefore, by Theorem 4.4 (ii), $\mu=\lambda_{0}$, the operator $L_{\lambda_{0}}$ is positive-critical, and for some constant $c$ we have $\varphi=c \phi$. In addition, part (iii) of Theorem 4.5 implies that $\left(\lambda_{0}-\lambda\right)^{-1}$ is the unique eigenvalue of $\left.\mathcal{G}_{\lambda}\right\rceil_{L^{p}}\left(\phi_{p}\right)$ with a nonnegative eigenfunction $\phi$ for all $1 \leq p<\infty$ and all $\lambda<\lambda_{0}$.

The simplicity of $\left(\lambda_{0}-\lambda\right)^{-1}$ as an eigenvalue of $\left.\mathcal{G}_{\lambda}\right|_{L^{p}\left(\phi_{p}\right)}$ for $2 \leq p \leq \infty$ follows now from the simplicity for $p=2$ and the embedding (2.14).

Next we study the case $p=1$, and obtain the simplicity of the eigenvalue $\left(\lambda_{0}-\lambda\right)^{-1}$ for all $p$ under the assumption that $(\mu-\lambda)^{-1}$ is an eigenvalue of $\mathcal{G}_{\lambda} \uparrow_{L^{1}(W \tilde{\phi})}$. Note that for $p=1$ the result does not depend on a particular positive solution $\phi$ of the equation $L_{\mu} u=0$ in $\Omega$ since $L^{1}\left(\phi_{1}\right)=L^{1}(W \tilde{\phi})$. As a result, we obtain $L^{1}$ - and $L^{p}$-Liouville theorems for solutions of the 
equation $L_{\mu} u=0$ which are eigenfunctions of the operator $\mathcal{G}_{\lambda}$ with an eigenvalue $(\mu-\lambda)^{-1}$.

Theorem 4.7. Let $L$ be an elliptic operator on $\Omega$ of the form (2.1), and let $W$ be a positive potential. Fix $\mu \leq \lambda_{0}$. Let $\tilde{\phi}$ be a positive solution of the equation $L_{\mu}^{\star} u=0$ in $\Omega$, For $\lambda<\mu$ consider the operator $\left.\mathcal{G}_{\lambda}\right\rceil_{L^{1}(W \tilde{\phi})}$. Suppose further that $(\mu-\lambda)^{-1}$ is an eigenvalue of $\mathcal{G}_{\lambda} \uparrow_{L^{1}(W \tilde{\phi})}$ with an eigenfunction $\varphi$.

(i) The eigenfunction $\varphi$ has a definite sign, $\mu=\lambda_{0}$, and $L_{\lambda_{0}}$ is positivecritical with respect to $W$ with a ground state $\varphi$. In particular, $\left(\lambda_{0}-\lambda\right)^{-1}$ is a simple eigenvalue of the operator $\left.\mathcal{G}_{\lambda}\right\rceil_{L^{1}(W \tilde{\phi})}$.

(ii) Set

$$
\varphi_{p}:=|\varphi|^{-1}(|\varphi| W \tilde{\phi})^{1 / p} \quad 1 \leq p \leq \infty .
$$

Then for all $\lambda<\lambda_{0}$ and all $1 \leq p \leq \infty, \varphi$ is an eigenfunction of $\mathcal{G}_{\lambda}\left\lceil_{L^{p}}\left(\varphi_{p}\right)\right.$ with an eigenvalue $\left(\lambda_{0}-\lambda\right)^{-1}$. Moreover, $\left(\lambda_{0}-\lambda\right)^{-1}$ is a simple eigenvalue, and it is the unique eigenvalue with a nonnegative eigenfunction.

Proof. (i) Fix any positive solution $\phi$ of the equation $L_{\mu} u=0$ in $\Omega$, and let $\phi_{p}:=\phi^{-1}(\phi W \tilde{\phi})^{1 / p}$. Clearly, $L^{1}\left(\phi_{1}\right)=L^{1}(W \tilde{\phi})$. By Theorem 3.1, for any $\lambda<\mu$ the operator $\mathcal{G}_{\left.\lambda\right|_{L^{1}(W \tilde{\phi})}}$ is bounded with a norm $\left\|\left.\mathcal{G}_{\lambda}\right|_{L^{1}(W \tilde{\phi})}\right\|_{L^{1}(W \tilde{\phi})} \leq$ $(\mu-\lambda)^{-1}$.

Let $\varphi$ be an eigenfunction of $\mathcal{G}_{\lambda} \uparrow_{L^{1}(W \tilde{\phi})}$ with an eigenvalue $(\mu-\lambda)^{-1}$. By our assumption,

$$
\frac{|\varphi(x)|}{\mu-\lambda}=\left|\int_{\Omega} G_{L_{\lambda}}^{\Omega}(x, y) W(y) \varphi(y) \mathrm{d} \nu(y)\right| \quad \forall x \in \Omega .
$$

Therefore, using (2.5) we obtain

$$
\begin{aligned}
& \frac{1}{\mu-\lambda} \int_{\Omega}|\varphi(x)| W(x) \tilde{\phi}(x) \mathrm{d} \nu= \\
& \quad \int_{\Omega}\left|\int_{\Omega} G_{L_{\lambda}}^{\Omega}(x, y) W(y) \varphi(y) \mathrm{d} \nu(y)\right| W(x) \tilde{\phi}(x) \mathrm{d} \nu(x) \leq \\
& \int_{\Omega}\left(\int_{\Omega} G_{L_{\lambda}}^{\Omega}(x, y) W(y)|\varphi(y)| \mathrm{d} \nu(y)\right) W(x) \tilde{\phi}(x) \mathrm{d} \nu(x)= \\
& \int_{\Omega}\left(\int_{\Omega} G_{L_{\lambda}}^{\Omega}(x, y) W(x) \tilde{\phi}(x) \mathrm{d} \nu(x)\right) W(y)|\varphi(y)| \mathrm{d} \nu(y) \leq \\
& \frac{1}{\mu-\lambda} \int_{\Omega} \tilde{\phi}(y) W(y)|\varphi(y)| \mathrm{d} \nu(y) .
\end{aligned}
$$


Therefore, the two inequalities in (4.7) are equalities, and in particular, for almost all $x \in \Omega$ we have

$$
\left|\int_{\Omega} G_{L_{\lambda}}^{\Omega}(x, y) W(y) \varphi(y) \mathrm{d} \nu(y)\right|=\int_{\Omega} G_{L_{\lambda}}^{\Omega}(x, y) W(y)|\varphi(y)| \mathrm{d} \nu(y),
$$

and hence $\varphi$ does not change its sign in $\Omega$. Moreover, the equality in (4.7) implies also that $\tilde{\phi}$ is an invariant solution of the equation $L_{\mu}^{\star} u=0$ in $\Omega$. Since $|\varphi| W \tilde{\phi} \in L^{1}(\Omega)$, Remark 2.3 implies that $\mu=\lambda_{0}, L_{\lambda_{0}}$ is positive-critical, and $|\varphi|$ is its ground state. Hence, the simplicity of the 'maximal' eigenvalue follows. Consequently, part (ii) follows using the embedding (2.14), part (i) of the present theorem, and part (iii) of Theorem 4.5.

Corollary 4.8. Let $L$ be an elliptic operator on $\Omega$ of the form (2.1), and let $W$ be a positive potential. Assume further that $L^{\star} \mathbf{1}=0$. For $\lambda<0$ consider the operator $\mathcal{G}_{\lambda}\left\lceil_{L^{1}(W)}\right.$.

Suppose that $|\lambda|^{-1}$ is an eigenvalue of $\left.\mathcal{G}_{\lambda}\right|_{L^{1}(W)}$ with an eigenfunction $\varphi$, and set $\varphi_{p}:=|\varphi|^{-1}(|\varphi| W)^{1 / p}$, where $1 \leq p \leq \infty$. Then $\varphi$ has a definite sign, $\lambda_{0}=0, L$ is positive-critical, and for all $\lambda<0$ and all $1 \leq p \leq \infty$, $|\lambda|^{-1}$ is the unique eigenvalue of $\left.\mathcal{G}_{\lambda}\right|_{L^{p}\left(\varphi_{p}\right)}$ with a nonnegative eigenfunction. Moreover, $|\lambda|^{-1}$ is a simple eigenvalue of $\mathcal{G}_{\lambda}\left\lceil_{L^{p}}\left(\varphi_{p}\right)\right.$

Example 4.9. Let $\Omega=\mathbb{R}^{d}$, and consider a uniformly elliptic operator $L$ with bounded smooth coefficients on $\mathbb{R}^{d}$ such that $L^{\star} \mathbf{1}=0$ in $\mathbb{R}^{d}$ (these conditions can be relaxed). For example, assume that $L$ is of the form

$$
L u:=-\operatorname{div}(A(x) \nabla u+u \tilde{b}(x)) \quad x \in \mathbb{R}^{d} .
$$

Suppose that the equation $L u=0$ in $\mathbb{R}^{d}$ admits a solution $\varphi$ satisfying $\varphi \in L^{1}\left(\mathbb{R}^{d}\right)$. Let $k_{L}^{\mathbb{R}^{d}}(x, y, t)$ the heat kernel associated with the operator $L$ on $\mathbb{R}^{d}$. Then

$$
v(x, t):=\int_{\mathbb{R}^{d}} k_{L}^{\mathbb{R}^{d}}(x, y, t) \varphi(y) \mathrm{d} y
$$

is a well defined $L^{1}$-solution of the Cauchy problem with the initial condition $\varphi$. Since the uniqueness of the Cauchy problem for $L^{1}$-initial conditions holds true [2, 3], it follows that $v=\varphi$. Fix $\lambda<0$. It follows that

$$
\begin{aligned}
\frac{\varphi(x)}{|\lambda|}=\int_{0}^{\infty} e^{\lambda t} v(x, t) \mathrm{d} t=\int_{0}^{\infty} e^{\lambda t}\left(\int_{\mathbb{R}^{d}} k_{L}^{\mathbb{R}^{d}}(x, y, t) \varphi(y) \mathrm{d} y\right) \mathrm{d} t= \\
\int_{\mathbb{R}^{d}}\left(\int_{0}^{\infty} e^{\lambda t} k_{L}^{\mathbb{R}^{d}}(x, y, t) \mathrm{d} t\right) \varphi(y) \mathrm{d} y=\int_{\mathbb{R}^{d}} G_{L_{\lambda}}^{\mathbb{R}^{d}}(x, y) \varphi(y) \mathrm{d} y .
\end{aligned}
$$

So, $\varphi$ is an eigenfunction of $\mathcal{G}_{\lambda} \uparrow_{L^{1}\left(\mathbb{R}^{d}\right)}$ with an eigenvalue $|\lambda|^{-1}$. Corollary 4.8 implies that $L$ is positive-critical with respect to $W=\mathbf{1}$, and $|\varphi|>0$ is the corresponding ground state. In particular, $\mathbf{1}$ is an invariant positive solution of the operator $L^{\star}$. Moreover, $|\lambda|^{-1}$ is a simple eigenvalue of $\left.\mathcal{G}_{\lambda}\right|_{L^{1}\left(\mathbb{R}^{d}\right)}$. 
Let $1 \leq p \leq \infty$, and $\varphi_{p}:=|\varphi|^{\frac{1}{p}-1}$. Then, by Corollary 4.8 for all $\lambda<0$ and all $1 \leq p \leq \infty,|\lambda|^{-1}$ is the unique eigenvalue of $\mathcal{G}_{\lambda} \uparrow_{L^{p}\left(\varphi_{p}\right)}$ with a nonnegative eigenfunction $|\varphi|$. Moreover, $|\lambda|^{-1}$ is a simple eigenvalue of $\mathcal{G}_{\lambda}\left\lceil_{L^{p}\left(\varphi_{p}\right)}\right.$ for all $1 \leq p \leq \infty$.

Remark 4.10. For $L^{p}$-Liouville theorems for symmetric diffusion operators on complete Riemannian manifolds, see [17, 36] and references therein.

\section{SEmigroups And Generators}

Definition 5.1. Let $B$ be a Banach space and $\Lambda \subset \mathbb{C}$, and consider a oneparameter family of operators $\mathcal{J}(\lambda) \in \mathcal{L}(B)$ defined for each $\lambda \in \Lambda$. The family $\{\mathcal{J}(\lambda) \mid \lambda \in \Lambda\}$ is called a pseudoresolvent if

$$
\mathcal{J}(\lambda)-\mathcal{J}(\nu)=(\nu-\lambda) \mathcal{J}(\lambda) \mathcal{J}(\nu)
$$

holds for all $\lambda, \nu \in \Lambda$ (see [13, Definition 4.3]).

Let $L$ be an elliptic operator on $\Omega$ of the form (2.1), and $W$ a positive potential. Fix $\mu \leq \lambda_{0}=\lambda_{0}(L, W, \Omega)$, and let

$$
\Lambda:= \begin{cases}\{\lambda \in \mathbb{R} \mid \lambda \leq \mu\} & \text { if } L_{\mu} \text { is subcritical, } \\ \{\lambda \in \mathbb{R} \mid \lambda<\mu\} & \text { if } \mu=\lambda_{0} \text { and } L_{\lambda_{0}} \text { is critical. }\end{cases}
$$

Recall that by (2.10) of [26], for all $\lambda, \nu \in \Lambda$ the corresponding Green functions satisfy the (pointwise) resolvent equation

$$
G_{L_{\lambda}}^{\Omega}(x, y)=G_{L_{\nu}}^{\Omega}(x, y)+(\lambda-\nu) \int_{\Omega} G_{L_{\lambda}}^{\Omega}(x, z) W(z) G_{L_{\nu}}^{\Omega}(z, y) \mathrm{d} \nu(z)
$$

for all $x, y \in \Omega, x \neq y$.

Let $\phi$ and $\tilde{\phi}$ be two fixed positive solutions of the equations $L_{\mu} u=0$ and $L_{\mu}^{\star} u=0$ in $\Omega$, respectively. It follows from Theorem 3.1 and (5.2) that for any $1 \leq p \leq \infty$, the family

$$
\left.\left\{\mathcal{G}_{(-\lambda)} \Upsilon_{L^{p}\left(\phi_{p}\right)} \mid-\lambda \in \Lambda\right\}=\left\{\mathcal{G}_{L+\lambda W}\right\rceil_{L^{p}\left(\phi_{p}\right)} \mid-\lambda \in \Lambda\right\}
$$

is a pseudoresolvent on $L^{p}\left(\phi_{p}\right)$.

We claim that for $1 \leq p<\infty$ and $\lambda \in \Lambda$, the range of $\mathcal{G}_{\lambda} \uparrow_{L^{p}}\left(\phi_{p}\right)$ is dense in $L^{p}\left(\phi_{p}\right)$. Indeed, take $u \in C_{0}^{\infty}(\Omega)$, and let $N_{u} \in \mathbb{N}$ be such that supp $u \Subset \Omega_{N_{u}}$. Set $f(x):=(W(x))^{-1} L_{\lambda} u(x)$. For $n \geq N_{u}$ denote $u_{n}:=\mathcal{G}_{\lambda}^{\Omega_{n}} \Gamma_{L^{p}}\left(\phi_{p}\right) f$. Clearly, supp $f \subset \Omega_{n}$ for all $n \geq N_{u}$. Therefore, by uniqueness, for any such $n$ we have $u_{n}=u$ in $\Omega_{n}$, and consequently, $u=\mathcal{G}_{\lambda}^{\Omega} \uparrow_{L^{p}}\left(\phi_{p}\right) f$, and $u$ belongs to the range of $\left.\mathcal{G}_{\lambda}\right\rceil_{L^{p}}\left(\phi_{p}\right)$. Since for $1 \leq p<\infty$ the space $C_{0}^{\infty}(\Omega)$ is dense in $L^{p}\left(\phi_{p}\right)$, it follows that the range of $\left.\mathcal{G}_{\lambda}\right|_{L^{p}\left(\phi_{p}\right)}$ is dense in $L^{p}\left(\phi_{p}\right)$.

On the other hand, by Theorem 4.2, zero is not an eigenvalue of the operators $\mathcal{G}_{\lambda} \uparrow_{L^{p}\left(\phi_{p}\right)}$ for $\lambda<\mu$ and all $1<p \leq \infty$. Moreover, for $1 \leq p \leq \infty$ we have

$$
\left\|\mathcal{G}_{\lambda}\right\|_{L^{p}\left(\phi_{p}\right)} \leq \frac{1}{\mu-\lambda}
$$


and in particular,

$$
\limsup _{\lambda \rightarrow-\infty}\left\|\lambda \mathcal{G}_{\lambda}\right\|_{L^{p}\left(\phi_{p}\right)} \leq \lim _{\lambda \rightarrow-\infty} \frac{-\lambda}{\mu-\lambda}=1 .
$$

Moreover, (5.3) implies also that if $\mu \geq 0$ and $\lambda<0$, then

$$
\left\|\lambda \mathcal{G}_{\lambda}\right\|_{L^{p}\left(\phi_{p}\right)} \leq 1 .
$$

Therefore, by Proposition III.4.6 and Corollary III.4.7 of [13], and the HilleYosida theorem [13, Theorem II.3.5], we have:

Theorem 5.2. Let $L$ be an elliptic operator on $\Omega$ of the form (2.1), and let $W$ be a positive potential. Fix $1<p<\infty, \mu \leq \lambda_{0}$, and let $\Lambda$ be as in (5.1). Let $\phi$ and $\tilde{\phi}$ be two fixed positive solutions of the equations $L_{\mu} u=0$ and $L_{\mu}^{\star} u=0$ in $\Omega$, respectively.

(i) The pseudoresolvent family

$$
\left\{\mathcal{G}_{(-\lambda)} \uparrow_{L^{p}\left(\phi_{p}\right)} \mid-\lambda \in \Lambda\right\}
$$

is a resolvent of a densely defined closed operator

$$
A_{p}:=-\left(\mathcal{G}_{\lambda_{1}}\left\lceil_{L^{p}\left(\phi_{p}\right)}\right)^{-1}-\lambda_{1} \quad \text { where } \lambda_{1} \in \Lambda\right.
$$

on $L^{p}\left(\phi_{p}\right)$ with a domain $D\left(A_{p}\right)=R\left(\mathcal{G}_{\lambda_{1}} \Upsilon_{L^{p}}\left(\phi_{p}\right)\right)$. In particular, $A_{p}=-\frac{1}{W} L$ on $C_{0}^{\infty}(\Omega)$. Moreover, $(-\mu, \infty) \subset \rho\left(A_{p}\right)$, and for $\lambda \in(-\mu, \infty)$ we have

$$
R\left(\lambda, A_{p}\right):=\left(\lambda-A_{p}\right)^{-1}=\mathcal{G}_{(-\lambda)} \Gamma_{L^{p}}\left(\phi_{p}\right) \cdot
$$

(ii) Zero is not an eigenvalue of $\left.\mathcal{G}_{\lambda}\right\rceil_{L^{p}}\left(\phi_{p}\right)$.

(iii) If $\mu \geq 0$, then $\left(A_{p}, D\left(A_{p}\right)\right)$ generates a strongly continuous contraction semigroup. Moreover, for every $\lambda \in \mathbb{C}$ with $\operatorname{Re} \lambda>0$ one has $\lambda \in \rho\left(A_{p}\right)$, and

$$
\left\|R\left(\lambda, A_{p}\right)\right\|_{L^{p}\left(\phi_{p}\right)} \leq \frac{1}{\operatorname{Re} \lambda} .
$$

\section{Compactness and semismall perturbations}

Throughout this section, we assume that $L$ is subcritical in $\Omega$ and $W>0$ is a semismall perturbation of $L$ and $L^{\star}$ in $\Omega$. By Remark 2.7, $\lambda_{0}>0$, and $L_{\lambda_{0}}$ is positive-critical. Denote by $\phi$ and $\tilde{\phi}$ the ground states of $L_{\lambda_{0}}$ and $L_{\lambda_{0}}^{\star}$, respectively. We may assume that $\phi\left(x_{0}\right)=1$. So,

$$
\left(L-\lambda_{0} W\right) \phi=\left(L^{\star}-\lambda_{0} W\right) \tilde{\phi}=0 \text { in } \Omega \text { and } \tilde{\phi} W \phi \in L^{1}(\Omega) .
$$

Without loss of generality, we may assume that

$$
\int_{\Omega} \tilde{\phi}(x) W(x) \phi(x) \mathrm{d} \nu(x)=1 .
$$

It follows that $\tilde{\phi} \in\left(L^{1}\left(\tilde{\phi}_{1}\right)\right) \subset\left(L^{\infty}\left(\phi_{\infty}\right)\right)^{\star}$, and for every $f \in L^{\infty}\left(\phi_{\infty}\right)$ we have $\langle\tilde{\phi}, f\rangle=\int_{\Omega} \tilde{\phi}(x) W(x) f(x) \mathrm{d} \nu$.

The aim of the present section is to prove that under the above assumptions, the integral operator $\mathcal{G}_{\lambda}$ is compact on $L^{p}\left(\phi_{p}\right)$ for any $1 \leq p \leq \infty$ and 
$\lambda<\lambda_{0}$, and its spectrum is $p$-independent. We first prove the compactness of $\mathcal{G}_{\lambda}$.

Theorem 6.1. Let $L$ be a subcritical operator in $\Omega$. Assume that $W>0$ is a semismall perturbation of $L^{\star}$ and $L$ in $\Omega$. Then for any $1 \leq p \leq \infty$ and $\lambda<\lambda_{0}$, the integral operators

$$
\begin{aligned}
& \mathcal{G}_{\lambda} f(x)=\int_{\Omega} G_{L-\lambda W}^{\Omega}(x, y) W(y) f(y) \mathrm{d} \nu(y), \\
& \mathcal{G}_{\lambda}^{\odot} f(y)=\int_{\Omega} G_{L-\lambda W}^{\Omega}(x, y) W(x) f(x) \mathrm{d} \nu(x)
\end{aligned}
$$

are compact on $L^{p}\left(\phi_{p}\right)$ and $L^{p}\left(\tilde{\phi}_{p}\right)$, respectively.

Proof. By [28, Theorem 5.1], the operators $\mathcal{G}_{\lambda} \uparrow_{L^{\infty}\left(\phi_{\infty}\right)}$ and $\mathcal{G}_{\lambda}^{\odot} \uparrow_{L^{\infty}\left(\tilde{\phi}_{\infty}\right)}$ are compact on $L^{\infty}\left(\phi_{\infty}\right)$ and $L^{\infty}\left(\tilde{\phi}_{\infty}\right)$, respectively. For the sake of completeness, we prove the compactness of $\mathcal{G}_{\lambda} \uparrow_{L^{\infty}\left(\phi_{\infty}\right)}$; the proof of the compactness of $\mathcal{G}_{\lambda}^{\odot} \uparrow_{L^{\infty}\left(\tilde{\phi}_{\infty}\right)}$ is identical.

Let $\left\{f_{n}\right\}$ be a bounded sequence in $L^{\infty}\left(\phi_{\infty}\right)$. By Theorem 3.1, the sequence $u_{n}:=\left.\mathcal{G}_{\lambda}\right|_{L^{\infty}\left(\phi_{\infty}\right)} f_{n}$ is bounded in $L^{\infty}\left(\phi_{\infty}\right)$, and satisfies

$$
\left|u_{n}(x)\right| \leq \int_{\Omega} G_{L_{\lambda}}^{\Omega}(x, y) W(y)\left|f_{n}(y)\right| \mathrm{d} \nu(y) \leq C \phi_{0}(x),
$$

where $C:=\left(\lambda_{0}-\lambda\right)^{-1} \sup _{n}\left\|f_{n}\right\|_{\infty, \phi_{\infty}}$ is independent of $n$. Moreover, it follows that $u_{n}$ is the unique function in $L^{\infty}\left(\phi_{\infty}\right)$ which is a (weak) solution of the equation $L_{\lambda} u=f_{n}$ in $\Omega$ (cf. [28, Theorem 4.6]). Consequently, a standard elliptic argument implies that the sequence $\left\{u_{n}\right\}$ admits a subsequence which converges in the compact open topology to a function $u$. Clearly, $\|u\|_{\infty, \phi_{\infty}} \leq C$, so, $u \in L^{\infty}\left(\phi_{\infty}\right)$

Since $W$ is a semismall perturbation, it follows that for any given $\varepsilon>0$ there exists $K$ such that for any $k \geq K$ and $n, m \in \mathbb{N}$

$$
\begin{aligned}
& \int_{\Omega_{k}^{*}} G_{L_{\lambda}}^{\Omega}(x, y) W(y)\left|f_{n}(y)-f_{m}(y)\right| \mathrm{d} \nu(y) \leq \\
& 2 C \int_{\Omega_{k}^{*}} G_{L_{\lambda}}^{\Omega}(x, y) W(y) \phi_{0}(y) \mathrm{d} \nu(y)<\varepsilon \phi_{0}(x) \quad \forall x \in \overline{\Omega_{k}^{*}},
\end{aligned}
$$

and by the generalized maximum principle in $\Omega_{k}$, (6.3) holds for any $x \in \Omega$.

The local uniform convergence of $\left\{u_{n}\right\}$ implies that there exists $N_{\varepsilon} \in \mathbb{N}$ such that $\left|u_{n}-u_{m}\right| \leq \varepsilon \phi_{0}$ in $\Omega_{K}$ for all $n, m \geq N_{\varepsilon}$.

Fix $n, m \geq N_{\varepsilon}$. It follows from [28, Lemma 4.3] and the linearity that on $\Omega_{K}^{*}$ we have

$$
u_{n}(x)-u_{m}(x)=h_{n, m}(x)+\int_{\Omega_{K}^{*}} G_{L_{\lambda}}^{\Omega_{K}^{*}}(x, y) W(y)\left(f_{n}(y)-f_{m}(y)\right) \mathrm{d} \nu(y),
$$


where $h_{n, m} \in L^{\infty}\left(\phi_{\infty}\right)$ satisfies

$$
L_{\lambda} h_{n, m}=0 \quad \text { in } \Omega_{K}^{*}, \quad \text { and } \quad h_{n, m}(x)=u_{n}(x)-u_{m}(x) \quad x \in \partial \Omega_{K} .
$$

Since $\left|h_{n, m}\right| \leq \varepsilon \phi_{0}$ on $\partial \Omega_{K}$, and $h_{n, m}$ has minimal growth in $\Omega$, it follows that $\left|h_{n, m}\right| \leq 2 \varepsilon \phi_{0}$ in $\Omega_{K}^{*}$. On the other hand, by (6.3) we have

$$
\begin{aligned}
& \left|\int_{\Omega_{K}^{*}} G_{L_{\lambda}}^{\Omega_{K}^{*}}(x, y) W(y)\left(f_{n}(y)-f_{m}(y)\right) \mathrm{d} \nu(y)\right| \leq \\
& \quad \int_{\Omega_{K}^{*}} G_{L_{\lambda}}^{\Omega}(x, y) W(y)\left|f_{n}(y)-f_{m}(y)\right| \mathrm{d} \nu(y)<\varepsilon \phi_{0}(x) \quad \forall x \in \Omega_{K}^{*} .
\end{aligned}
$$

Consequently, we infer that $\left|u_{n}-u_{m}\right| \leq 3 \varepsilon \phi_{0}$ in $\Omega_{K}^{*}$ for all $n, m \geq N_{\varepsilon}$. Thus, $u_{n} \rightarrow u$ in $L^{\infty}\left(\phi_{\infty}\right)$.

Since for each $1 \leq p \leq \infty$ the operator $\mathcal{G}_{\lambda}\left\lceil_{L^{p}\left(\phi_{p}\right)}\right.$ is bounded on $L^{p}\left(\phi_{p}\right)$, and $\mathcal{G}_{\lambda}\left\lceil_{L^{\infty}\left(\phi_{\infty}\right)}\right.$ is compact on $L^{\infty}\left(\phi_{\infty}\right)$, it follows from a variant of the Riesz-Thorin interpolation theorem with respect to compact operators [8, Theorem 1.1] that $\left.\mathcal{G}_{\lambda}\right\rceil_{L^{p}\left(\phi_{p}\right)}$ and are compact for all $1 \leq p \leq \infty$. The same is true for $\mathcal{G}_{\lambda}^{\odot} \uparrow_{L^{p}}\left(\tilde{\phi}_{p}\right)$.

Remark 6.2. For $\lambda<\lambda_{0}$ the operators $\mathcal{G}_{\lambda} \uparrow_{L^{\infty}\left(\phi_{\infty}\right)}$ and $\mathcal{G}_{\lambda}^{\odot} \uparrow_{L^{\infty}\left(\tilde{\phi}_{\infty}\right)}$ are the dual operators of $\mathcal{G}_{\lambda}^{\odot} \uparrow_{L^{1}\left(\tilde{\phi}_{1}\right)}$ and $\mathcal{G}_{\lambda}\left\lceil_{L^{1}\left(\phi_{1}\right)}\right.$, respectively. Therefore, the well-known Schauder theorem directly implies that $\mathcal{G}_{\lambda}^{\odot} \uparrow_{L^{1}\left(\tilde{\phi}_{1}\right)}$ and $\left.\mathcal{G}_{\lambda}\right\rceil_{L^{1}\left(\phi_{1}\right)}$ are compact on $L^{1}\left(\tilde{\phi}_{1}\right)$ and $L^{1}\left(\phi_{1}\right)$, respectively.

Remark 6.3. In the proof of Theorem 6.1 we used the fact that real interpolation preserves the compactness of an operator. We recall that in his remarkable paper [7] A. Calderón implicitly asked a question which is apparently still open today: Does complex interpolation preserve the compactness of an operator? For a recent survey on this question see [9].

The next theorem discusses the spectral properties of $\left.\mathcal{G}_{\lambda}\right\rceil_{L^{p}}\left(\phi_{p}\right)$.

Theorem 6.4. Under the assumptions of Theorem 6.1] we have:

(1) For $1 \leq p \leq \infty$, the spectrum of $\mathcal{G}_{\lambda}\left\lceil_{L^{p}\left(\phi_{p}\right)}\right.$ contains 0 , and besides, consists of at most a sequence of eigenvalues of finite multiplicity which has no point of accumulation except 0 .

(2) For any $1 \leq p \leq \infty, \phi$ (resp. $\tilde{\phi})$ is the unique nonnegative eigenfunction of the operator $\left.\mathcal{G}_{\lambda}\right\rceil_{L^{p}\left(\phi_{p}\right)}$ (resp., $\left.\mathcal{G}_{\lambda}^{\odot} \uparrow_{L^{p}\left(\tilde{\phi}_{p}\right)}\right)$. The corresponding eigenvalue $\nu=\left(\lambda_{0}-\lambda\right)^{-1}$ is simple.

(3) The spectrum of $\left.\mathcal{G}_{\lambda}\right\rceil_{L^{p}}\left(\phi_{p}\right)$ is $p$-independent for all $1 \leq p \leq \infty$, and we have

$$
0 \in \sigma\left(\mathcal{G}_{\lambda}\left\lceil_{L^{p}\left(\phi_{p}\right)}\right)=\sigma\left(\mathcal{G}_{\lambda}^{\odot} \uparrow_{L^{p}\left(\tilde{\phi}_{p}\right)}\right) \subset \overline{B\left(0,\left(\lambda_{0}-\lambda\right)^{-1}\right)} .\right.
$$


Proof. (1) The characterization of the spectrum of $\mathcal{G}_{\lambda}\left\lceil_{L^{p}}\left(\phi_{p}\right)\right.$ for each $p$ follows from the Riesz-Schauder theory for compact operators.

(2) Follows from Theorem 4.7.

(3) The compactness of all the operators $\mathcal{G}_{\lambda}\left\lceil_{L^{p}\left(\phi_{p}\right)}\right.$ implies that it is enough to show that $\sigma_{\text {point }}\left(\mathcal{G}_{\lambda} \uparrow_{L^{p}\left(\phi_{p}\right)}\right)$ is $p$-independent.

By (2.14) we have for any $1 \leq p \leq \infty$ that

$$
\left.\sigma_{\text {point }}\left(\mathcal{G}_{\lambda}\right\rceil_{L^{\infty}\left(\phi_{\infty}\right)}\right) \subset \sigma_{\text {point }}\left(\mathcal { G } _ { \lambda } \lceil _ { L ^ { p } ( \phi _ { p } ) } ) \subset \sigma _ { \text { point } } \left(\mathcal{G}_{\lambda}\left\lceil_{L^{1}\left(\phi_{1}\right)}\right),\right.\right.
$$

and

$$
\sigma_{\text {point }}\left(\mathcal{G}_{\lambda}^{\odot} \uparrow_{L^{\infty}}\left(\tilde{\phi}_{\infty}\right)\right) \subset \sigma_{\text {point }}\left(\mathcal{G}_{\lambda}^{\odot} \uparrow_{L^{p}\left(\tilde{\phi}_{p}\right)}\right) \subset \sigma_{\text {point }}\left(\mathcal{G}_{\lambda}^{\odot} \uparrow_{L^{1}\left(\tilde{\phi}_{1}\right)}\right) .
$$

On the other hand, $\phi$ and $\tilde{\phi}$ are $\lambda_{0}$-invariant positive solutions of the operator $L$ and $L^{\star}$, respectively. Therefore, Theorem 4.4 implies that $\left\|\mathcal{G}_{\lambda}\right\|_{L^{p}\left(\phi_{p}\right)}=$ $(\mu-\lambda)^{-1}$.

Recall that by Theorem 3.1, for any $1 \leq p<\infty$, the operator $\mathcal{G}_{\lambda}^{\odot} \uparrow_{L^{p^{\prime}}}\left(\tilde{\phi}_{p^{\prime}}\right)$ is the dual operator of $\mathcal{G}_{\lambda} \uparrow_{L^{p}\left(\phi_{p}\right)}$, and $\mathcal{G}_{\lambda} \uparrow_{L^{p^{\prime}}\left(\phi_{p^{\prime}}\right)}$ is the dual of $\mathcal{G}_{\lambda}^{\odot} \uparrow_{L^{p}\left(\tilde{\phi}_{p}\right)}$. Since the spectra of a bounded operator and its dual are equal, we have

$$
\left.\left.\sigma\left(\mathcal{G}_{\lambda}\right\rceil_{L^{1}\left(\phi_{1}\right)}\right)=\sigma\left(\mathcal{G}_{\lambda}^{\odot} \uparrow_{L^{\infty}\left(\tilde{\phi}_{\infty}\right)}\right), \quad \sigma\left(\mathcal{G}_{\lambda}^{\odot} \uparrow_{L^{1}\left(\tilde{\phi}_{1}\right)}\right)=\sigma\left(\mathcal{G}_{\lambda}\right\rceil_{L^{\infty}\left(\phi_{\infty}\right)}\right) .
$$

Thus all the point-spectra in (6.4) and (6.5) are equal.

\section{REFERENCES}

[1] S. Agmon, Bounds on exponential decay of eigenfunctions of Schrödinger operators. In Schrödinger Operators (Como, 1984), Lecture Notes in Math. 1159, Springer, Berlin, 1985, 1-38.

[2] D.G. Aronson and P. Besala, Uniqueness of solutions of the Cauchy problem for parabolic equations. J. Math. Anal. Appl. 13 (1966), 516-526.

[3] D.G. Aronson and P. Besala, Corrections to "Uniqueness of solutions of the Cauchy problem for parabolic equations", J. Math. Anal. Appl. 17 (1967), 194-196.

[4] W. Arendt, Gaussian estimates and interpolation of the spectrum in $L^{p}$. Differential Integral Equations 7 (1994), 1153-1168.

[5] R. Bauñelos and B. Davis, Heat kernel, eigenfunctions, and conditioned Brownian motion in planar domains. J. Funct. Anal. 84 (1989), 188-200.

[6] A. Ben Amor and W. Hansen, Continuity of eigenvalues for Schrödinger operators, $L^{p}$-properties of Kato type integral operators. Math. Ann. 321 (2001), 925-953.

[7] A. P. Calderón, Intermediate spaces and interpolation, the complex method, Studia Math. 24 (1964), 113-190.

[8] M. Cwikel, Real and complex interpolation and extrapolation of compact operators. Duke Math. J. 65 (1992), 333-343.

[9] M. Cwikel, Nigel Kalton and complex interpolation of compact operators. arXiv: 1410.4527.

[10] E. B. Davies, $L^{p}$ spectral independence and $L^{1}$ analyticity. J. London Math. Soc. (2) 52 (1995), 177-184.

[11] E. B. Davies, $L^{p}$ spectral independence for certain uniformly elliptic operators. In Partial differential equations and mathematical physics (Copenhagen, 1995; Lund, 1995), Progr. Nonlinear Differential Equations Appl. 21, Birkhäuser Boston, Boston, MA, 1996, 122-125. 
[12] E. B. Davies and B. Simon, Ultracontractivity and the heat kernel for Schrödinger operators and Dirichlet Laplacians. J. Funct. Anal. 59 (1984), 335-395.

[13] K-J. Engel and R. Nagel, One-parameter semigroups for linear evolution equations. Graduate Texts in Mathematics 194. Springer-Verlag, New York, 2000.

[14] R. Killip, M. Visan and X. Zhang, Riesz transforms outside a convex obstacle. Int. Math. Res. Not. (2015), 47 pages. doi:10.1093/imrn/rnv338.

[15] P. C. Kunstmann and H. Vogt, Weighted norm estimates and $L_{p}$-spectral independence of linear operators. Colloq. Math. 109 (2007), 129-146.

[16] D. Lenz, P. Stollmann and D. Wingert, Compactness of Schrödinger semigroups. Math. Nachr. 283 (2010), 94-103.

[17] X. D. Li, Liouville theorems for symmetric diffusion operators on complete Riemannian manifolds. J. Math. Pures Appl. (9) 84 (2005), 1295-1361.

[18] V. Liskevich and H. Vogt, On $L^{p}$-spectra and essential spectra of second-order elliptic operators. Proc. London Math. Soc. (3) 80 (2000), 590-610.

[19] V. Liskevich, Z. Sobol and H. Vogt, On the $L_{p}$-theory of $C_{0}$-semigroups associated with second-order elliptic operators. II. J. Funct. Anal. 193 (2002), 55-76.

[20] G. Metafune, D. Pallara and M. Wacker, Compactness properties of Feller semigroups. Studia Math. 153 (2002), 179-206.

[21] M. Murata, Structure of positive solutions to $(-\Delta+V) u=0$ in $\mathbb{R}^{n}$. Duke Math. J. 53 (1986), 869-943.

[22] M. Murata, Semismall perturbations in the Martin theory for elliptic equations. Israel J. Math. 102 (1997), 29-60.

[23] M. Murata, Integral representations of nonnegative solutions for parabolic equations and elliptic Martin boundaries. J. Funct. Anal. 245 (2007), 177-212.

[24] Y. Pinchover, On positive solutions of second-order elliptic equations, stability results and classification. Duke Math. J. 57 (1988), 955-980.

[25] Y. Pinchover, Criticality and ground states for second-order elliptic equations. J. Differential Equations 80 (1989), 237-250.

[26] Y. Pinchover, Criticality and ground states for second-order elliptic equations, II. J. Differential Equations 87 (1990), 353-364.

[27] Y. Pinchover, On nonexistence of any $\lambda_{0}$-invariant positive harmonic function, a counterexample to Stroock's conjecture. Comm. Partial Differential Equations 20 (1995), 1831-1846.

[28] Y. Pinchover, Maximum and anti-maximum principles and eigenfunctions estimates via perturbation theory of positive solutions of elliptic equations. Math. Ann. 314 (1999), 555-590.

[29] Y. Pinchover, Liouville-type theorem for Schrödinger operators. Comm. Math. Phys. 272 (2007), 75-84.

[30] M. Reed and B. Simon, Methods of modern mathematical physics IV. Analysis of operators. Academic Press, New York-London, 1978.

[31] Yu. A. Semenov, Stability of $L^{p}$-spectrum of generalized Schrödinger operators and equivalence of Green's functions. Internat. Math. Res. Notices 12 (1997), 573-593.

[32] H. Shindoh, Kernel estimates and $L^{p}$-spectral independence of generators of $C_{0^{-}}$ semigroups. J. Funct. Anal. 255 (2008), 1273-1295.

[33] B. Simon, Brownian motion, $L^{p}$ properties of Schrödinger operators and the localization of binding. J. Funct. Anal. 35 (1980), 215-229.

[34] Z. Sobol and H. Vogt, On the $L_{p}$-theory of $C_{0}$-semigroups associated with secondorder elliptic operators, I. J. Funct. Anal. 193 (2002), 24-54.

[35] E. M. Stein, Interpolation of linear operators. Trans. Amer. Math. Soc. 83 (1956), 482-492.

[36] J.-Y. Wu, $L^{p}$-Liouville theorems on complete smooth metric measure spaces, Bull. Sci. Math. 138 (2014), 510-539. 
Yehuda Pinchover, Department of Mathematics, Technion - Israel Institute of Technology, Haifa 32000, IsRael

E-mail address: pincho@techunix.technion.ac.il 